\title{
Analysis of neutron capture elements in metal-poor stars ${ }^{\star}$
}

\author{
T. V. Mishenina ${ }^{1,2}$ and V. V. Kovtyukh ${ }^{1,2}$ \\ 1 Astronomical observatory, Odessa State University, Shevchenko Park, 65014, Odessa, Ukraine \\ ${ }^{2}$ Isaac Newton Institute of Chile, Odessa Branch
}

Received 16 November 2000 / Accepted 7 February 2001

\begin{abstract}
We derived model atmosphere parameters $\left(T_{\text {eff }}, \log g,[\mathrm{Fe} / \mathrm{H}], V_{\mathrm{t}}\right)$ for 90 metal-deficient stars $(-0.5<[\mathrm{Fe} / \mathrm{H}]<-3)$, using echelle spectra from the ELODIE library (Soubiran et al. 1998). These parameters were analyzed and compared with current determinations by other authors. The study of the following elements was carried out: $\mathrm{Mg}, \mathrm{Si}, \mathrm{Ca}, \mathrm{Sr}, \mathrm{Y}, \mathrm{Ba}, \mathrm{La}, \mathrm{Ce}, \mathrm{Nd}$, and $\mathrm{Eu}$. The relative contributions of $s$ - and $r$-processes were evaluated and interpreted through theoretical computations of the chemical evolution of the Galaxy. The chemical evolution models (Pagel \& Tautvaišienè 1995; Timmes et al. 1995) depict quite well the behaviour of $[\mathrm{Si} / \mathrm{Fe}],[\mathrm{Ca} / \mathrm{Fe}]$ with $[\mathrm{Fe} / \mathrm{H}]$. The trend of $[\mathrm{Mg} / \mathrm{Fe}]$ compares more favourably with the computations of Pagel \& Tautvaišienè (1995) than those of Timmes et al. (1995). The runs of $n$-capture elements vs. metallicity are described well both by the model of Pagel \& Tautvaišienè $(1995,1997)$ and by the model of Travaglio et al. (1999) at $[\mathrm{Fe} / \mathrm{H}]>-1.5$, when the matter of the Galaxy is sufficiently homogeneous. The analysis of $n$-capture element abundances confirms the jump in $[\mathrm{Ba} / \mathrm{Fe}]$ at $[\mathrm{Fe} / \mathrm{H}]=-2.5$. Some stars from our sample at $[\mathrm{Fe} / \mathrm{H}]<-2.0$ show a large scatter of $\mathrm{Sr}, \mathrm{Ba}, \mathrm{Y}, \mathrm{Ce}$. This scatter is not caused by the errors in the measurements, and may reflect the inhomogeneous nature of the prestellar medium at early stages of galactic evolution. The matching of $[\mathrm{Ba} / \mathrm{Fe}]$, $[\mathrm{Eu} / \mathrm{Fe}]$ vs. $[\mathrm{Fe} / \mathrm{H}]$ with the inhomogeneous model by Travaglio et al. (2001a) suggests that at $[\mathrm{Fe} / \mathrm{H}]<-2.5$, the essential contribution to the $n$-rich element abundances derives from the $r$-process. The main sources of these processes may be low mass SN II. The larger dispersion of $s$-process element abundances with respect to $\alpha$-rich elements may arise both from the birth of metal-poor stars in globular clusters with following different evolutionary paths and (or) from differences in $s$-element enrichment in Galaxy populations.
\end{abstract}

Key words. nucleosynthesis, abundances - stars: abundances - stars: late-type - Galaxy: evolution

\section{Introduction}

The abundances of neutron-capture elements in metaldeficient stars provide an important clue to the chemical evolution and early enrichment of the Galaxy. Two main mechanisms are responsible for the production of these elements: the $r$-process (for "rapid" neutron capture) and the $s$-process (for "slow" neutron capture), depending on the intensity of the neutron flux available (Burbidge et al. 1957). In general, the $r$-and $s$-process syntheses are supposed to occur at different stages of a star's lifetime. The $r$-process nuclei are synthesized in massive stars that explode as Type II supernovae (SNe) (Cowan et al. 1991). The $s$-process is traditionally divided into two types: the weak $s$-component and the main $s$-component. The weak $s$-component is responsible for the production of lighter elements (Sr, Y, Zr) during core-He burning and shellC burning in massive stars (Lamb et al. 1977; Raiteri

Send offprint requests to: T. V. Mishenina,

e-mail: tamar@deneb.odessa.ua

* Based on spectra collected at the Observatoire de HauteProvence (OHP), France et al. 1991, 1992). The main $s$-component elements (heavier than Sr) can be synthesized during the thermal pulses in the AGB phase of intermediate- and low-mass stars (Iben \& Renzini 1982; Hollowell \& Iben 1989).

In some works concerning $n$-capture element determinations (Spite \& Spite 1978; Gilroy et al. 1988), the enrichments relative to iron have been found not to follow that of $\alpha$ - or iron-peak elements. Truran (1981) speculated that, for low metallicity stars, this might be caused by the dominant role of the $r$-process. Later studies considered both the $r$-, and $s$-process contributions in metaldeficient stars (Gratton \& Sneden 1994; McWilliam et al. 1995; Ryan et al. 1996). Gratton \& Sneden (1994) discovered that the relative contribution of the $s$-process is smaller in metal-poor stars than in the solar system, but that it is not negligible, even in stars as metal-poor as $[\mathrm{Fe} / \mathrm{H}]=-2.5$. McWilliam et al. (1995) confirmed a rapid increase for $[\mathrm{Sr} / \mathrm{Fe}],[\mathrm{Ba} / \mathrm{Fe}]$ vs. $[\mathrm{Fe} / \mathrm{H}]$ at a unique metallicity, about $[\mathrm{Fe} / \mathrm{H}]=-2.4$, that earlier had been detected by Spite \& Spite (1978). This observation implies that a distinct phase of nucleosynthesis occurred 
before the Galaxy reached $[\mathrm{Fe} / \mathrm{H}]=-2.4$. McWilliam et al. (1995) also found that the dispersions in some heavy element abundances ( $\mathrm{Sr}$ for example) represent a scatter in the original stellar compositions at $[\mathrm{Fe} / \mathrm{H}]<-2.5$. Ryan et al. (1996) showed that $[\mathrm{Sr} / \mathrm{Fe}]$ exhibits a spread larger than $2 \operatorname{dex}$ at $[\mathrm{Fe} / \mathrm{H}]<-3$, while $[\mathrm{Ba} / \mathrm{Fe}]$ shows a scatter lower than that of $[\mathrm{Sr} / \mathrm{Fe}]$. The above-mentioned facts may have arisen from the weak $s$-process in massive stars or by $r$-processing. Investigation of more metal-deficient stars (McWilliam 1998; Sneden et al. 1998) confirmed the significant scatter in $n$-capture species at low metallicities. Burris et al. (2000) also found that the $n$-abundances show clear evidence for a large star-to-star dispersion at low metallicity. They confirmed that at $[\mathrm{Fe} / \mathrm{H}]<-2.4$, the abundance pattern of the heavy $n$-capture elements is well-matched to a scaled Solar System $r$-process nucleosynthesis and that the contributions from the $s$-process can first be seen in some stars with metallicities as low as $[\mathrm{Fe} / \mathrm{H}] \sim-2.75$, and are present in most stars with metallicities $[\mathrm{Fe} / \mathrm{H}]>-2.3$.

The $n$-capture element enrichment of the Galaxy has been considered in several chemical evolution models (Pagel \& Tautvaišienė 1995, 1997; Travaglio et al. 1999). The inhomogeneous enrichment of the Galaxy has been studied by Raiteri et al. (1999), Tsujimoto et al. (2000), and Travaglio et al. (2001a).

The theory of galactic chemical evolution (Pagel \& Tautvaišiene 1995, 1997) predicts enrichment by $r$-process elements (Eu, Th) from type II supernovae and implies two separate time scales for $s$-process elements from AGB stars - of the order of $37 \mathrm{Myr}$ and $2.7 \mathrm{Gyr}$, corresponding to progenitor masses of about $8 M_{\odot}$ and $1.5 M_{\odot}$, respectively. This analytical model adopted a scheme of gas infall, the effect of which is a dilution of the metallicity of the interstellar matter at irregular intervals. This could provide an observational scatter in abundances of the order of 0.15 dex.

The influence of AGB stars with various masses on the chemical enrichment of the Galaxy has been studied in some recent works (for example Travaglio et al. 1999; Gallino et al. 2000; Travaglio et al. 2001b). According to model of Travaglio et al. (1999), stellar yields for $n$ rich nuclei have been separated into their $s$-process and $r$ process components. The $s$-process-component yields proceed from AGB stars with masses $2-4 M_{\odot}$ and depend on stellar metallicity; the $r$-process is a primary process, possibly occurring in low mass SNII progenitors $\left(8-10 M_{\odot}\right)$. Lately, numerical techniques based on $N$-body/smooth particle hydrodynamic codes have been used to investigate galactic chemical evolution, in particular, Ba enrichment in the work of Raiteri et al. (1999). They showed that two Ba sources (low mass SNII, via a primary $r$-process and low mass AGB stars) can explain the Ba behaviour at different $[\mathrm{Fe} / \mathrm{H}]$ values.

One of the proposed inhomogeneous chemical evolution models of the Galaxy is the Monte Carlo model by Travaglio et al. (2001a). It is based on the idea of fragmentation and coalescence between interstellar gas clouds, taking into account the effects of local enrichment and mixing of the halo gas, and is focussed on elements like $\mathrm{Eu}$, produced by $r$-process from relatively low mass SN and $\mathrm{Ba}$ and $\mathrm{Sr}$, for which the $s$-process contribution is larger. In the case of Eu production from high-mass SN $\left(15-25 M_{\odot}\right)$, the time delay in the enrichment of $\mathrm{Eu}$ with respect to Fe would be too small to explain the observed spread in $[\mathrm{Eu} / \mathrm{Fe}]$ at $-3.5<[\mathrm{Fe} / \mathrm{H}]<-2.5$.

The important role of the neutron capture elements in chemical evolution theories was emphasized by the investigation of abundances in mildly metal-poor stars $([\mathrm{Fe} / \mathrm{H}] \sim-1)$ by Jehin et al. (1999). They have suggested a scenario of formation of metal-poor stars based on two distinct phases of chemical enrichment. The first phase essentially consists of supernova explosions of massive stars and the second of contributions from stellar winds of intermediate mass stars. So, Jehin et al. (1999) assume that all thick disk and field halo stars were born in globular clusters, from which they escaped, either during an early disruption of the cluster (Population IIa) or, later, through an evaporation process (Population IIb).

Recent studies of the neutron-capture-element production in our Galaxy have made noticeable progress, but uncertainties still remain. First of all, there is the amount of scatter in $s$ - and $r$-element abundances. For this, various explanations exist for example, the diffusion of stellar orbits (Fuchs et al. 1994). The use of homogeneous observational high-dispersion data for a large sample of objects and a uniform approach in the data reduction and in computations allows us to exclude scatter due to observational and modeling errors and permits us to test theories of galactic chemical evolution more confidently.

To understand the role of $n$-capture element abundances in the chemical evolution of the Galaxy we had to address the following points: 1) Analysis of the main parameters of metal-poor stars, begining with the effective temperatures $T_{\text {eff }}$ and the surface gravities $\log g ; 2$ ) Examination of the contributions from $r$-, s-process in $n$ capture element abundances on the basis of extensive stellar observational data; 3) Comparison of the $\alpha$-, $n$-capture element abundances with the prediction of the theories of galactic chemical evolution (Timmes et al. 1995; Pagel \& Tautvaišienè 1995, 1997; Travaglio et al. 1999; Travaglio et al. 2001a); 4) Investigation of the presence of two subpopulations among metal-poor stars, revealed by Jehin et al. (1999).

\section{Observations and data reduction}

90 target stars (Table 1) were selected from a library of high-resolution spectra (Soubiran et al. 1998) according to the criteria of $-0.5<[\mathrm{Fe} / \mathrm{H}]<-3$. These values of $[\mathrm{Fe} / \mathrm{H}]$ correspond to the stars that belong to the thick disk and halo. The diagram of $M_{\mathrm{bol}}$ vs. $b-y$ for program stars is presented in Fig. 1. $B-V$ and $b-y$ were taken from the SIMBAD database; $M_{\mathrm{bol}}$-bolometric magnitudes are from Soubiran et al. (1998). 
Table 1. Photometrical data and atmosphere parameters for program stars

\begin{tabular}{|c|c|c|c|c|c|c|c|c|}
\hline Star & $V$ & $B-V$ & $b-y$ & $M_{\text {bol }}$ & $T_{\text {eff }}$ & $\log g$ & $V_{\mathrm{t}}$ & {$[\mathrm{Fe} / \mathrm{H}]$} \\
\hline HD 245 & 8.41 & 0.65 & - & 4.29 & 5400 & 3.4 & 0.5 & -0.78 \\
\hline HD 2796 & 8.48 & 0.75 & 0.542 & - & 4900 & 1.6 & 1.5 & -2.21 \\
\hline HD 3546 & 4.34 & 0.87 & 0.553 & 0.51 & 4950 & 2.1 & 1.5 & -0.63 \\
\hline HD 3567 & 9.25 & 0.46 & 0.328 & 4.04 & 5950 & 3.8 & 0.5 & -1.20 \\
\hline HD 4306 & 9.02 & 0.73 & 0.530 & 2.15 & 5000 & 2.1 & 1.5 & -2.52 \\
\hline HD 5395 & 4.62 & 0.96 & 0.590 & 0.30 & 4800 & 2.5 & 1.0 & -0.19 \\
\hline HD 5916 & 6.86 & 0.90 & 0.565 & 0.63 & 4863 & 1.7 & 1.2 & -0.51 \\
\hline HD 6582 & 5.17 & 0.69 & 0.437 & 5.60 & 5240 & 4.2 & 0.2 & -0.89 \\
\hline HD 6755 & 7.72 & 0.72 & 0.487 & 1.94 & 5100 & 2.7 & 1.2 & -1.47 \\
\hline HD 6833 & 6.75 & 1.18 & 0.753 & -0.52 & 4400 & 1.0 & 1.5 & -0.89 \\
\hline HD 8724 & 8.30 & 0.96 & 0.686 & - & 4600 & 1.5 & 1.5 & -1.65 \\
\hline HD 10700 & 3.49 & 0.72 & 0.443 & 5.51 & 5270 & 4.2 & 0.5 & -0.56 \\
\hline HD 13530 & 5.31 & 0.92 & - & 1.05 & 4750 & 2.5 & 1.0 & -0.48 \\
\hline HD 13783 & 8.30 & 0.68 & 0.420 & 5.21 & 5350 & 4.1 & 0.5 & -0.61 \\
\hline HD 15596 & 6.21 & 0.90 & 0.580 & 0.96 & 4750 & 2.5 & 1.0 & -0.67 \\
\hline HD 18768 & 6.72 & 0.59 & 0.388 & 3.30 & 5700 & 3.5 & 1.0 & -0.51 \\
\hline HD 19445 & 8.04 & 0.46 & 0.351 & 4.96 & 6000 & 4.0 & 1.5 & -1.89 \\
\hline HD 23439 & 7.67 & 0.78 & 0.487 & 5.51 & 5100 & 4.3 & 1.0 & -1.14 \\
\hline HD 25329 & 8.51 & 0.88 & 0.529 & 6.87 & 4850 & 4.25 & 1.5 & -1.73 \\
\hline HD 26297 & 7.46 & - & 0.737 & - & 4300 & 0.5 & 1.7 & -1.91 \\
\hline HD 37828 & 6.86 & 1.14 & 0.726 & -0.66 & 4350 & 1.0 & 1.5 & -1.49 \\
\hline HD 44007 & 8.05 & 0.84 & 0.559 & 1.36 & 4950 & 2.25 & 1.5 & -1.49 \\
\hline HD 45282 & 8.00 & 0.66 & 0.451 & 2.15 & 5350 & 3.4 & 1.3 & -1.28 \\
\hline HD 46480 & 5.94 & 0.89 & 0.546 & 1.98 & 4800 & 2.7 & 0.8 & -0.49 \\
\hline HD 51530 & 6.20 & 0.45 & 0.345 & 2.76 & 6100 & 3.8 & 0.8 & -0.39 \\
\hline HD 63791 & 7.89 & - & 0.612 & - & 4625 & 1.75 & 1.2 & -1.67 \\
\hline HD 64090 & 8.27 & 0.61 & 0.430 & 5.83 & 5400 & 4.3 & 1.5 & -1.69 \\
\hline HD 64606 & 7.43 & 0.83 & 0.452 & 5.82 & 5250 & 4.0 & 0.5 & -0.82 \\
\hline HD 76932 & 5.80 & 0.53 & 0.359 & 4.06 & 5840 & 4.0 & 1.0 & -0.90 \\
\hline HD 84937 & 8.33 & 0.40 & 0.302 & 3.68 & 6250 & 3.8 & 1.5 & -2.00 \\
\hline HD 87140 & 8.97 & 0.70 & 0.480 & 1.95 & 5100 & 2.5 & 1.5 & -1.71 \\
\hline HD 88609 & 8.59 & 0.93 & 0.683 & - & 4600 & 1.0 & 1.5 & -2.66 \\
\hline HD 88725 & 7.75 & 0.60 & 0.397 & 4.85 & 5650 & 4.3 & 1.0 & -0.65 \\
\hline HD 94028 & 8.21 & 0.47 & 0.344 & 4.51 & 5950 & 4.0 & 1.5 & -1.43 \\
\hline HD 103095 & 6.42 & 0.75 & 0.484 & 6.37 & 5000 & 4.4 & 0.3 & -1.39 \\
\hline HD 105755 & 8.59 & - & 0.384 & 4.01 & 5800 & 3.85 & 1.5 & -0.65 \\
\hline HD 108076 & 8.03 & 0.56 & 0.386 & 5.07 & 5700 & 4.35 & 0.7 & -0.85 \\
\hline HD 108317 & 8.03 & - & 0.449 & 1.06 & 5250 & 2.4 & 1.7 & -2.17 \\
\hline HD 110184 & 8.27 & 1.17 & 0.818 & - & 4380 & 0.6 & 1.9 & -2.27 \\
\hline HD 114762 & 7.30 & 0.53 & 0.365 & 4.16 & 5800 & 4.0 & 1.0 & -0.72 \\
\hline HD 117876 & 6.11 & 0.96 & - & -0.01 & 4750 & 2.25 & 1.4 & -0.47 \\
\hline HD 122563 & 6.18 & 0.90 & 0.639 & -1.30 & 4570 & 1.1 & 1.2 & -2.42 \\
\hline HD 122956 & 7.22 & 1.01 & 0.668 & -0.54 & 4635 & 1.5 & 1.5 & -1.60 \\
\hline HD 124897 & -0.05 & 1.23 & 0.753 & -0.83 & 4350 & 1.6 & 1.6 & -0.58 \\
\hline HD 127243 & 5.58 & 0.83 & 0.544 & 0.45 & 5000 & 2.25 & 1.4 & -0.65 \\
\hline HD 132142 & 7.77 & 0.79 & 0.479 & 5.67 & 5100 & 4.0 & 1.0 & -0.51 \\
\hline HD 134169 & 7.67 & 0.55 & 0.370 & 3.69 & 5800 & 3.9 & 1.1 & -0.72 \\
\hline HD 140283 & 7.20 & 0.49 & 0.379 & 3.23 & 5650 & 3.5 & 1.2 & -2.50 \\
\hline HD 150177 & 6.33 & 0.48 & 0.334 & 3.07 & 6025 & 3.8 & 1.1 & -0.64 \\
\hline HD 157089 & 6.95 & 0.60 & 0.380 & 3.92 & 5785 & 3.8 & 1.0 & -0.56 \\
\hline HD 159482 & 8.37 & 0.57 & 0.384 & 4.84 & 5620 & 4.0 & 0.8 & -0.86 \\
\hline HD 160693 & 8.39 & 0.58 & 0.383 & 4.61 & 5750 & 4.0 & 1.0 & -0.46 \\
\hline HD 165195 & 7.31 & 1.29 & 0.919 & - & 4470 & 1.1 & 1.9 & -2.03 \\
\hline HD 165908 & 5.05 & 0.52 & 0.356 & 4.00 & 5925 & 4.1 & 1.1 & -0.61 \\
\hline HD 166161 & 8.12 & 0.97 & 0.685 & - & 5250 & 2.0 & 1.7 & -1.20 \\
\hline HD 175305 & 7.18 & 0.75 & 0.504 & 0.86 & 5050 & 2.3 & 1.4 & -1.42 \\
\hline HD 184499 & 6.62 & 0.58 & 0.390 & 4.00 & 5750 & 4.0 & 1.5 & -0.64 \\
\hline
\end{tabular}


Table 1. continued

\begin{tabular}{|c|c|c|c|c|c|c|c|c|}
\hline $\mathrm{HD} / \mathrm{BD}$ & $V$ & $B-V$ & $b-y$ & $M_{\mathrm{bol}}$ & $T_{\text {eff }}$ & $\log g$ & $V_{\mathrm{t}}$ & {$[\mathrm{Fe} / \mathrm{H}]$} \\
\hline HD 187111 & 7.71 & 1.22 & 0.830 & - & 4250 & 0.7 & 1.7 & -1.74 \\
\hline HD 188510 & 8.83 & 0.58 & 0.416 & 5.68 & 5500 & 4.4 & 1.5 & -1.48 \\
\hline HD 189558 & 7.72 & 0.57 & 0.386 & 3.45 & 5785 & 3.85 & 1.5 & -1.00 \\
\hline HD 194598 & 8.33 & 0.49 & 0.342 & 4.49 & 5890 & 4.0 & 1.5 & -1.16 \\
\hline HD 195633 & 8.54 & 0.53 & 0.361 & 3.14 & 6000 & 3.8 & 1.1 & -0.55 \\
\hline HD 201889 & 8.06 & 0.58 & 0.388 & 4.21 & 5600 & 4.1 & 1.2 & -0.85 \\
\hline HD 201891 & 7.37 & 0.51 & 0.358 & 4.52 & 5850 & 4.45 & 1.0 & -0.99 \\
\hline HD 204155 & 8.49 & 0.58 & 0.378 & 3.94 & 5600 & 3.8 & 1.0 & -0.78 \\
\hline HD 204543 & 8.28 & - & 0.635 & - & 4620 & 1.1 & 1.7 & -1.79 \\
\hline HD 208906 & 6.95 & 0.51 & 0.343 & 4.53 & 5965 & 4.2 & 1.7 & -0.71 \\
\hline HD 216143 & 7.80 & - & 0.688 & - & 4500 & 1.0 & 1.6 & -2.11 \\
\hline HD 216174 & 5.43 & 1.175 & - & -0.36 & 4400 & 1.9 & 1.5 & -0.56 \\
\hline HD 218502 & 8.25 & - & 0.312 & 3.91 & 6300 & 3.75 & 1.5 & -1.72 \\
\hline HD 218857 & 8.94 & 0.71 & 0.501 & - & 5050 & 2.4 & 1.2 & -1.84 \\
\hline HD 219617 & 8.16 & 0.49 & 0.344 & 3.45 & 5870 & 4.0 & 1.5 & -1.43 \\
\hline HD 221170 & 7.67 & 1.08 & 0.747 & - & 4500 & 1.0 & 1.5 & -2.05 \\
\hline HD 221377 & 7.56 & 0.39 & 0.296 & 2.67 & 6000 & 3.4 & 1.2 & -0.88 \\
\hline HD 224930 & 5.80 & 0.67 & 0.428 & 5.17 & 5300 & 4.1 & 0.2 & -0.85 \\
\hline HD 338529 & 9.37 & 0.39 & 0.300 & 3.44 & 6170 & 3.7 & 1.0 & -2.31 \\
\hline HD 345957 & 8.89 & 0.51 & 0.372 & -1.51 & 5800 & 3.7 & 1.0 & -1.33 \\
\hline $\mathrm{BD}-185550$ & 9.28 & 0.92 & 0.685 & - & 4600 & 0.5 & 1.2 & -3.01 \\
\hline $\mathrm{BD}+023375$ & 9.94 & 0.44 & 0.352 & 4.40 & 5960 & 3.6 & 0.5 & -2.25 \\
\hline $\mathrm{BD}+024651$ & 10.21 & 0.42 & 0.339 & - & 6000 & 3.5 & 1.0 & -1.82 \\
\hline $\mathrm{BD}+044551$ & 9.59 & 0.51 & 0.362 & - & 5750 & 3.5 & 0.9 & -1.51 \\
\hline $\mathrm{BD}+174708$ & 9.46 & 0.44 & 0.329 & 3.97 & 6000 & 4.0 & 0.7 & -1.56 \\
\hline $\mathrm{BD}+233130$ & 8.94 & - & - & 1.84 & 5100 & 2.25 & 1.0 & -2.62 \\
\hline $\mathrm{BD}+290366$ & 8.76 & 0.575 & 0.390 & 4.87 & 5600 & 4.2 & 0.9 & -1.01 \\
\hline $\mathrm{BD}+292091$ & 10.26 & 0.50 & 0.392 & 5.23 & 5850 & 4.2 & 1.6 & -1.03 \\
\hline $\mathrm{BD}+302611$ & 9.13 & 1.24 & 0.810 & - & 4300 & 0.7 & 1.6 & -1.41 \\
\hline $\mathrm{BD}+362165$ & 9.77 & 0.43 & 0.319 & 4.22 & 6175 & 4.2 & 0.8 & -1.51 \\
\hline $\mathrm{BD}+413931$ & 10.28 & - & - & 5.88 & 5450 & 4.6 & 1.0 & -1.68 \\
\hline $\mathrm{BD}+423607$ & 10.11 & - & - & 5.37 & 5850 & 4.0 & 0.9 & -1.97 \\
\hline $\mathrm{BD}+660268$ & 9.91 & 0.66 & 0.451 & 5.95 & 5350 & 4.5 & 1.0 & -1.95 \\
\hline
\end{tabular}

The spectra of the library were obtained with the echelle spectrograph ELODIE attached to the $1.93 \mathrm{~m}$ telescope at the Observatoire de Haute-Provence. They cover the spectral range $4400-6800 \AA$, the resolving power was $R=42000, \mathrm{~S} / \mathrm{N}$ was about 100 or higher. The spectra were straightened, cleaned from cosmic ray hits, bad pixels and telluric lines (Katz et al. 1998). The continuum level tracing, wavelength calibration and equivalent width $(E W)$ measurements were carried out by us using the DECH20 code (Galazutdinov 1992). Equivalent widths of the lines were measured by Gaussian function fitting.

The accuracy of the equivalent widths was estimated by comparing them to some independent measurements by other authors: the mean difference between $E W$ of HD 76932 by Jehin et al. (1999) and us is ( $E W(\mathrm{Jeh})-$ $E W($ our $))=-2.72(\sigma= \pm 2.8)$ for 55 lines; for the
Mg I line (5711 $\AA)$ in 18 common stars with Pilachowski et al. (1996), this difference is $E W($ Pil $)-E W($ our $)=-0.12$ ( $\sigma= \pm 3.2$ ). The comparison of the equivalent widths is shown in Figs. 2 and 3.

\section{Atmospheric parameters}

The choice of the right effective temperature $T_{\text {eff }}$ and the surface gravity $\log g$ is very important for the chemical composition of metal-poor stars. As one can see from the literature (Cayrel de Strobel et al. 1997), the $T_{\text {eff }}$ values obtained by different authors demonstrate considerable scatter - up to 400-600 K, - that generates uncertainties in abundances as large as 0.5 dex and more. A striking example of this is the use of a high temperature scale (King 1993) to resolve the problem of discrepancy between 
T. V. Mishenina and V. V. Kovtyukh: Analysis of $n$-capture elements in metal-poor stars

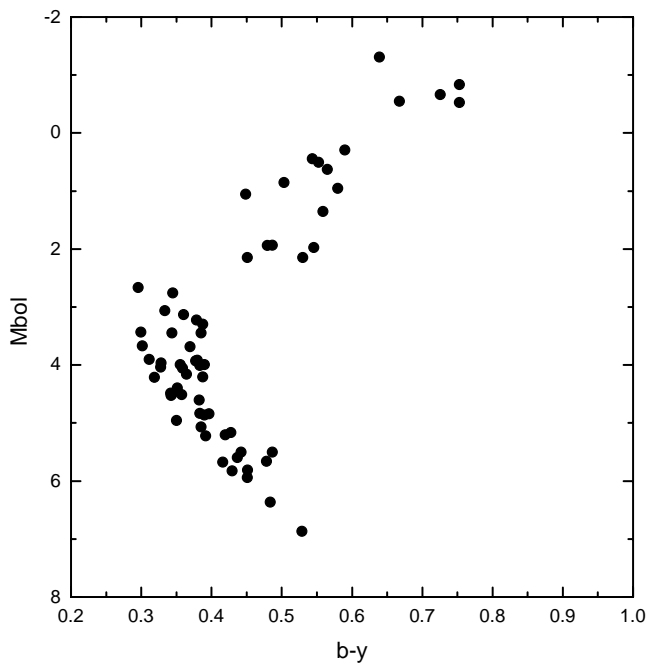

Fig. 1. The diagram of $M_{\text {bol }}$ vs. $b-y$ for program stars

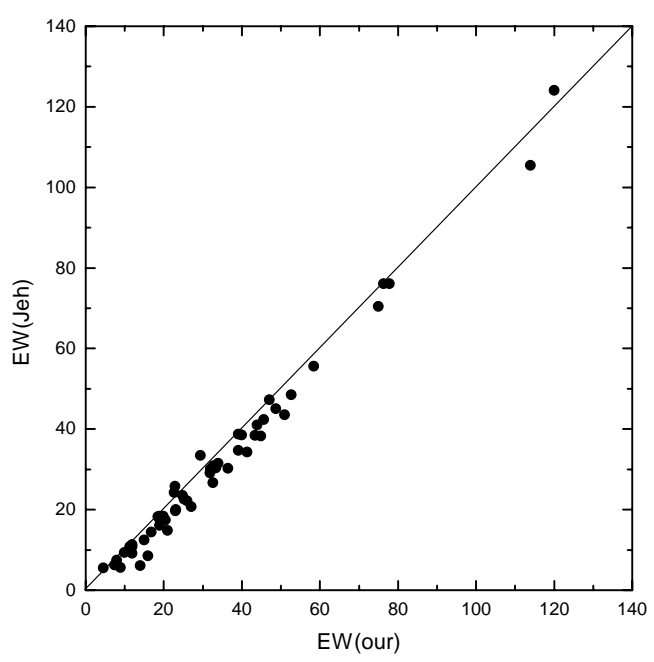

Fig. 2. The comparison of the equivalent widths measured in this study with those from the literature (Jehin et al. 1999)

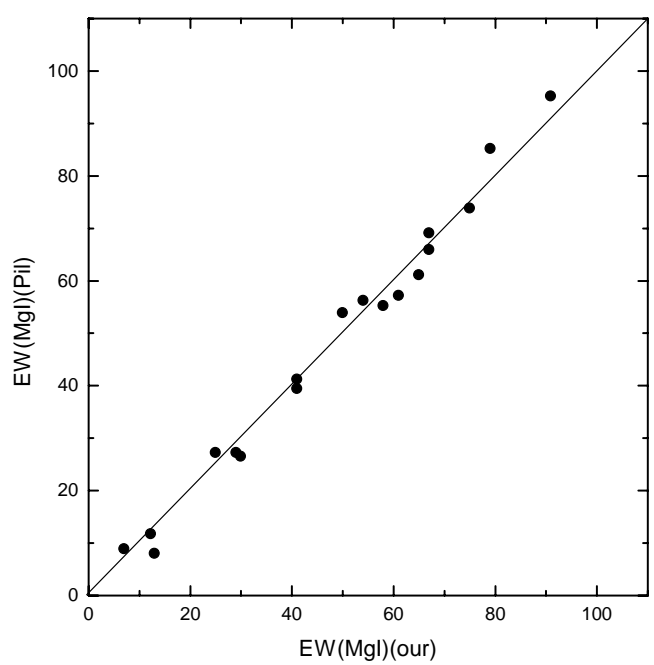

Fig. 3. The comparison of the equivalent widths measured in this study with those from literature (Pilachowski et al. 1996)

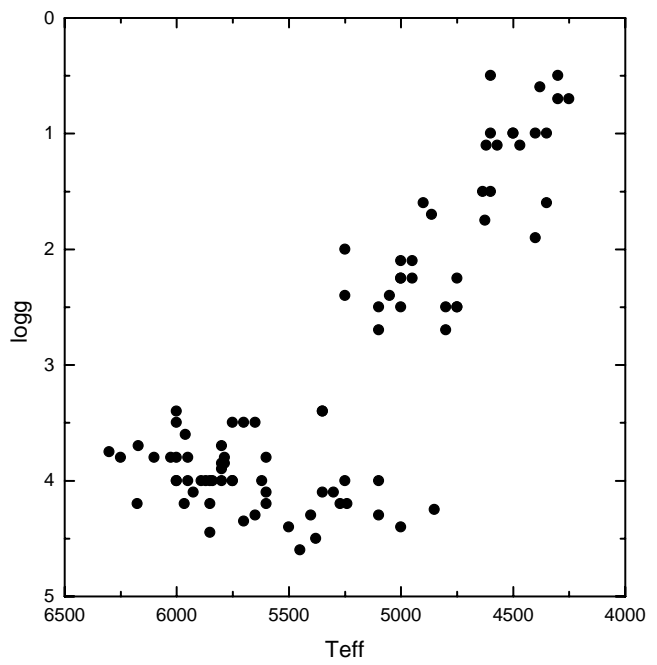

Fig. 4. The relations between the obtained characteristics of our target stars in this work: $\log g$ vs. $T_{\text {eff }}$

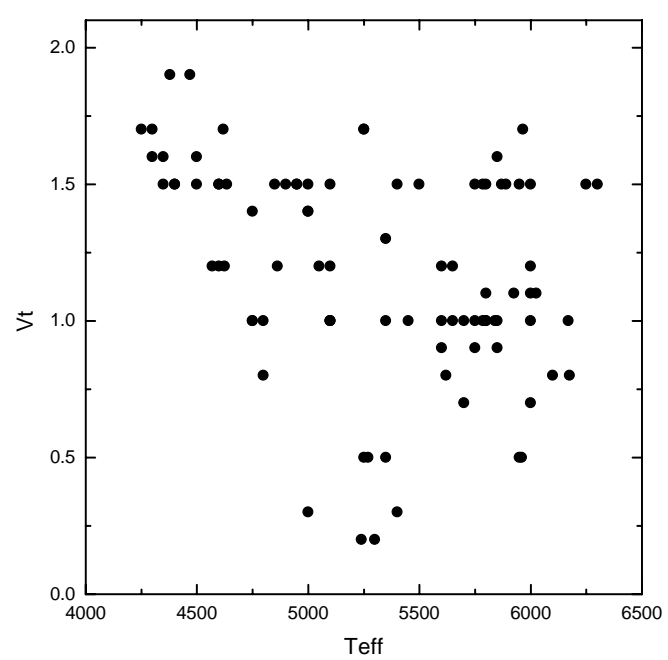

Fig. 5. The relation between the characteristics obtained our target stars: $V_{\mathrm{t}}$ vs. $T_{\text {eff }}$

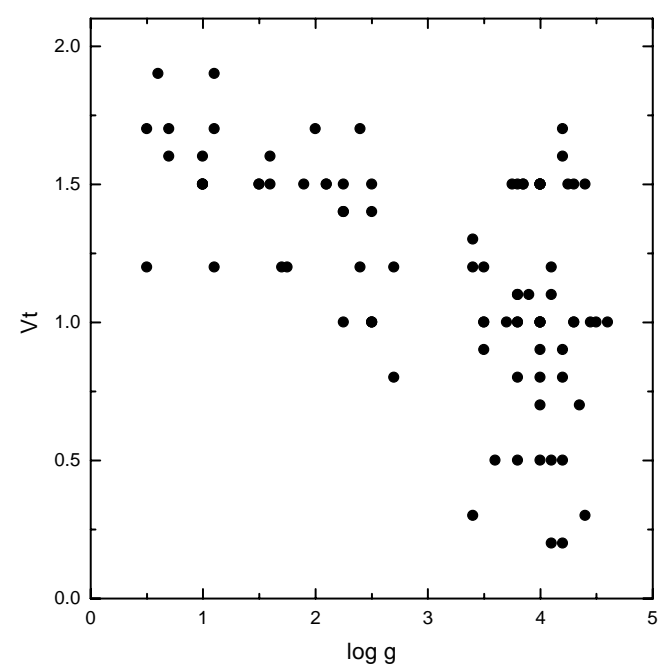

Fig. 6. The relation between the characteristics obtained our target stars: $V_{\mathrm{t}}$ vs. $\log g$ 


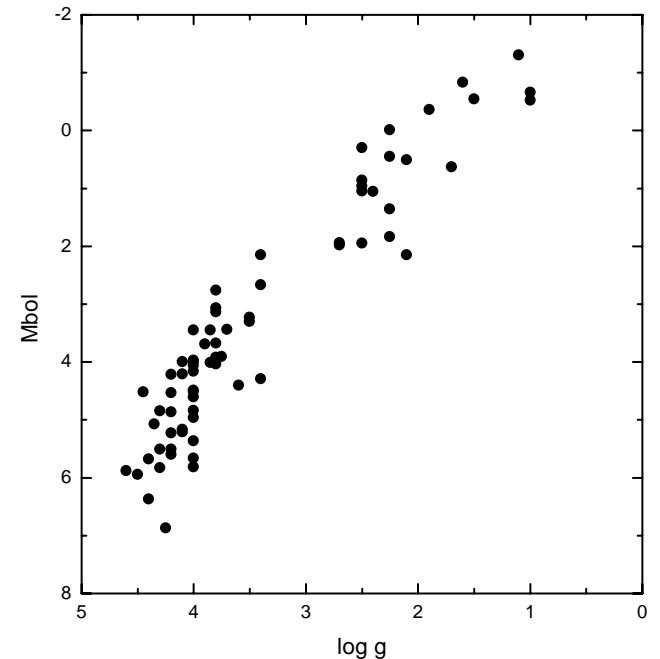

Fig. 7. The relation between the characteristics obtained our target stars: $M_{\text {bol }}$ vs. $\log g$

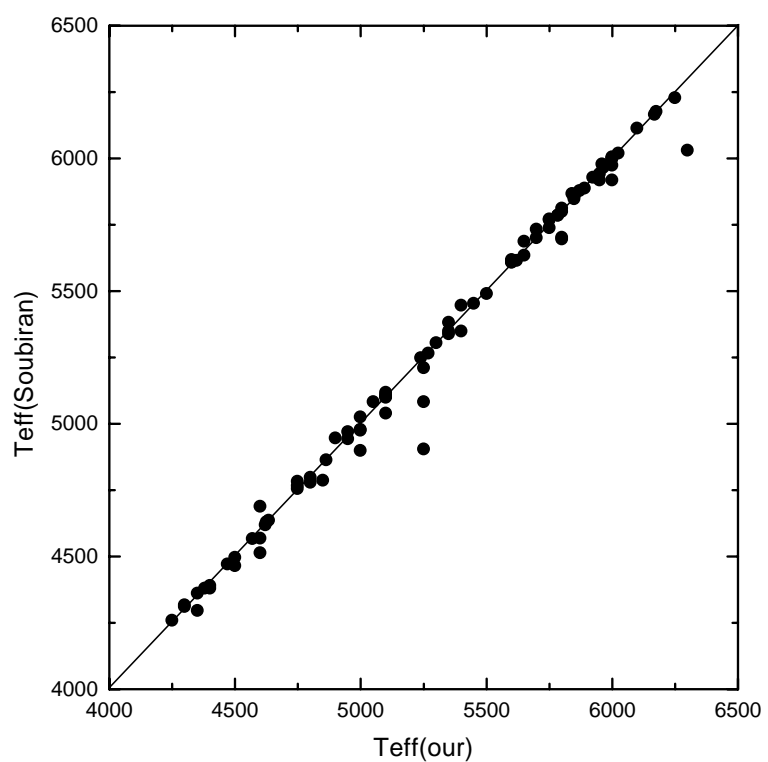

Fig. 8. The comparison of the adopted $T_{\text {eff }}$ with those derived by Soubiran et al. (1998)

oxygen abundances obtained from the forbidden and permitted O I lines. Recently, some new methods to derive more accurate $T_{\text {eff }}$ values have been developed, namely: a) the semi-direct Infrared Flux method (Alonso et al. 1996b, 1999a); b) Balmer line-profile fitting (Fuhrmann et al. 1994; Axer et al. 1995); c) new calibrations of $T_{\text {eff }}-$ photometric indices relations (Alonso et al. 1996a, 1999b; Gratton et al. 1996). These approaches provide the accuracy of $T_{\text {eff }}$ determinations at about $\pm 100 \mathrm{~K}$ or better.

The effective temperatures of the program stars were determined from the $\mathrm{H}_{\alpha}$ line--wing fitting. The far wings of $\mathrm{H}_{\alpha}$ are independent from gravity, metallicity and convection of the atmosphere model (Gratton et al. 1996). We used this approach for stars with $T_{\text {eff }}>4800 \mathrm{~K}$. At lower $T_{\text {eff }}$ (for cool stars) the $\mathrm{H}_{\alpha}$ line profile becomes very narrow, the wing extent is small and it results in lower

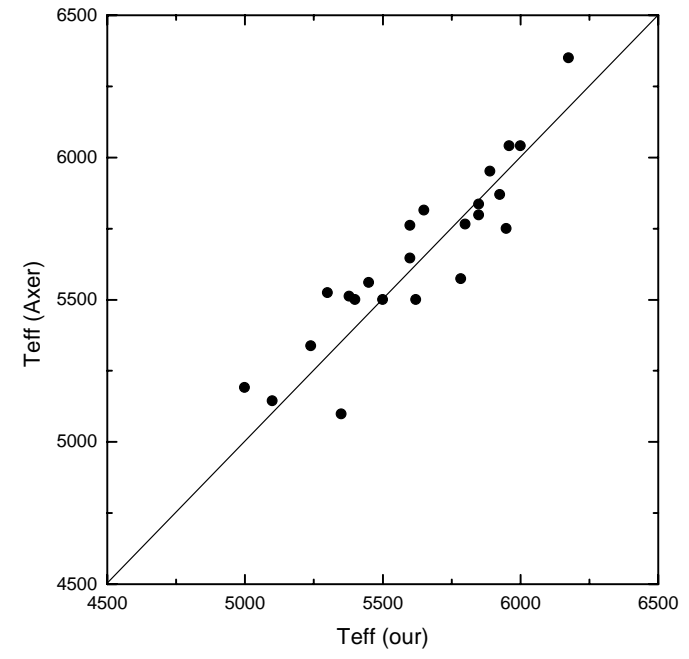

Fig. 9. The comparison of the adopted $T_{\text {eff }}$ with those derived by Axer et al. (1995)

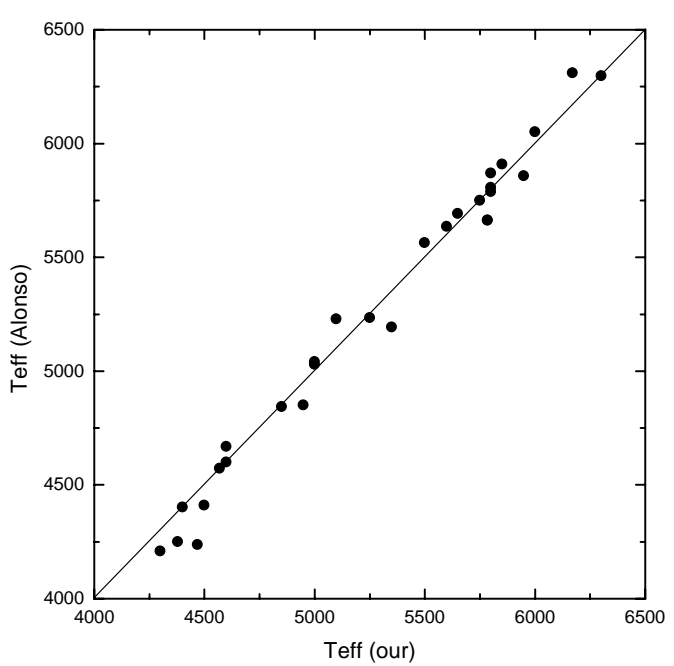

Fig. 10. The comparison of the adopted $T_{\text {eff }}$ with those derived by Alonso et al. (1996a, 1996b)

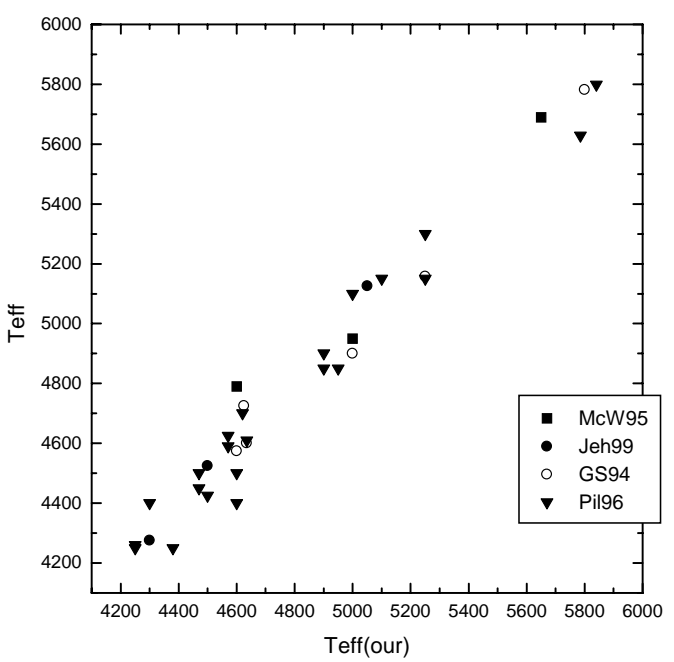

Fig. 11. The comparison of the adopted $T_{\text {eff }}$ with those derived by Gratton \& Sneden (1994), McWilliam et al. (1995), Pilachowski et al. (1996) and Jehin et al. (1999) 


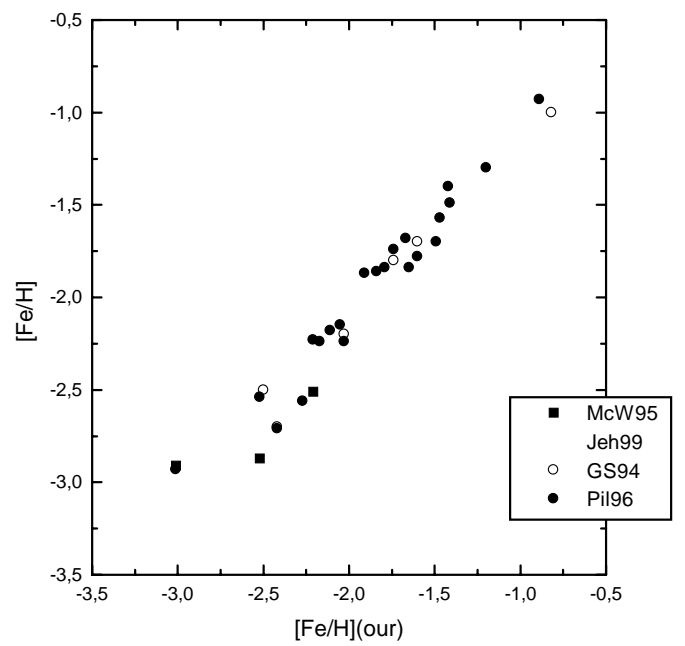

Fig. 12. The comparison of the adopted $[\mathrm{Fe} / \mathrm{H}]$ with those derived by Gratton \& Sneden (1994), McWilliam et al. (1995), Pilachowski et al. (1996) and Jehin et al. (1999)

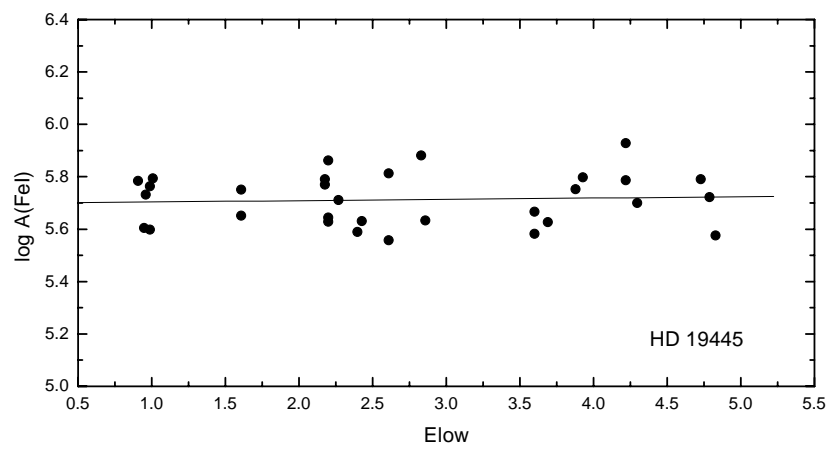

Fig. 13. Fe abundances as a function of exitation potencial $E_{\text {low }}$ for HD 19445

accuracy of $T_{\text {eff }}$ determinations. Synthetic profiles of the $\mathrm{H}_{\alpha}$ lines were computed by the STARSP code (Tsymbal 1996). As a first approximation, for $T_{\text {eff }}$ we took the values of Soubiran et al. (1998). For stars with $T_{\text {eff }}<4800 \mathrm{~K}, T_{\text {eff }}$ were determined under the condition that the abundances obtained from individual Fe I lines must be independent of the excitation potential $E_{\text {low }}$. Both methods (based on $\mathrm{H}_{\alpha}$ and on Fe I lines) are not dependent on photometric indices, calibrations, interstellar reddening and take into account the individual characteristics of a star's atmosphere.

The gravity has been determined by forcing Fe II to yield the same total iron abundance as Fe I. Microturbulent velocities $V_{\mathrm{t}}$ were determined by forcing the abundances determined from individual Fe I lines to be independent of equivalent width. Starting with $V_{\mathrm{t}}=$ $1.5 \mathrm{~km} \mathrm{~s}^{-1}$, we varied it until abundances computed for Fe I lines $(20 \mathrm{~m} \AA<E W<150 \mathrm{~m} \AA)$ and plotted as a function of $E W$-s showed Zero slope. The precision of the microturbulent velocity determination is $0.2 \mathrm{~km} \mathrm{~s}^{-1}$. Corresponding estimates for $T_{\text {eff }}$ and $\log g$ are $\pm 100 \mathrm{~K}$ and \pm 0.3 dex, respectively.

The adopted parameters of the program stars are given in Table 1. The relations between the obtained characteristics are represented in Figs. 4-7. Figure 4 exhibits the presence of dwarfs, subgiants and giants among our target objects. The $V_{\mathrm{t}}-\log g$ diagram (Fig. 6) shows that a distinct relation for giant stars exists $(0<\log g<3)$.

The $M_{\mathrm{bol}}-\log g$ diagram (Fig. 7) reflects the wellknown connection between the surface gravity of the star and its luminosity. To check the adopted model atmosphere parameters, we compared our values for $T_{\text {eff }}$ with those derived by Soubiran et al. (1998), Axer et al. (1995), Alonso et al. (1996a, 1996b), Gratton \& Sneden (1994), McWilliam et al. (1995), Pilachowski et al. (1996) and Jehin et al. (1999). See Figs. 8 to 11 for the comparisons. We also made comparisons for $\log g,[\mathrm{Fe} / \mathrm{H}]$ and $V_{\mathrm{t}}$ (for example, see Fig. 12). The mean differences between data obtained by us and by other authors are given in Table 2 . On the whole, the agreement is close and within the errors of determinations $(<1 \sigma)$. In Fig. $8\left(T_{\text {eff }}(\right.$ Sub $)-T_{\text {eff }}($ our $\left.)\right)$, three stars HD 108317, HD 166161, HD 218502 drop out from the average dependence $\left(\Delta T_{\text {eff }}=-167,-315,-270 \mathrm{~K}\right.$, respectively), but do not show any slope between the iron abundance determined from Fe I lines and its excitation potential $E_{\text {low }}$.

The values of $\Delta \log g$ are higher with respect to $\sigma$ than those of $T_{\text {eff }}$. Various interpretations of this effect are possible. One of them is non-LTE effects in the atmospheres of metal-poor stars (see Thévenin \& Idiart 1999 and also below).

Although the values of $\Delta \log g$ are within the determination errors, we estimated that it could have an effect on the abundance derivations. The uncertainty of 0.18 dex in $\log g$ corresponds to 0.05 dex in abundances determined from singly ionized species and it is negligible $(<0.01 \mathrm{dex})$ in the case of neutral species. It should be noted that the combined effects of even larger differences between the values of $T_{\text {eff }}$ and $\log g$ from Axer et al. (1995) and ours lead to changes in $[\mathrm{Fe} / \mathrm{H}]$ of only 0.02 dex.

\section{Abundance determination}

We selected about 150 unblended lines of Fe I, Fe II, Mg I, Si I, Ca I, Sr I, Y I, Ba II, La II, Ce II, Nd II and Eu II. The list of lines and atomic line data for $n$-capture elements are given in Table 3 . Unblended lines were identified by calculating the synthetic spectrum for the observed wavelength region using the STARSP code (Tsymbal 1996). In our analysis, we did not use the lines with $E W>150 \mathrm{~m} \AA$ to avoid the problems arising from the fact that a significant fraction of the flux in these lines originates in high layers, where the model atmospheres are less reliable, especially for low temperatures $\left(T_{\text {eff }}<4500 \mathrm{~K}\right)$.

An elemental abundance analysis of the program stars was carried out using the WIDTH-9 code by Kurucz, with Kurucz's model grids (1993) and oscillator strengths $\log g f(\mathrm{G}, \mathrm{K})$ from the paper of Gurtovenko \& Kostyk (1989). Oscillator strengths from this source for some of the elements (including $\mathrm{Ba}, \mathrm{Eu}$ ) allow for the effect of hyperfine structure. The required radiative and collisional damping constants are from Kurucz (1993). Appropriate models for each star were derived by means of standard 
Table 2. Parameter comparison

\begin{tabular}{rrccccrcc}
\hline$\Delta T_{\text {eff }}$ & $\sigma$ & $\Delta \log g$ & $\sigma$ & $\Delta V_{\mathrm{t}}$ & $\sigma$ & $\Delta[\mathrm{Fe} / \mathrm{H}]$ & $\sigma$ & Sources \\
\hline-14 & 82 & 0.07 & 0.31 & 0.16 & 0.40 & -0.10 & 0.11 & 1 \\
-14 & 58 & 0.18 & 0.24 & - & - & -0.06 & 0.15 & 2 \\
26 & 129 & 0.16 & 0.30 & - & - & 0.01 & 0.15 & 3 \\
-16 & 87 & - & - & - & - & - & - & 4 \\
\hline
\end{tabular}

* 1 - McWilliam et al. (1995), Jehin et al. (1999), Gratton \& Sneden (1994), Pilachowski et al. (1996); 2 - Soubiran et al. (1998); 3 - Axer et al. (1995); 4 - Alonso et al. (1996a, 1996b, 1999a, 1999b).

Table 3. The list of $n$-element lines used

\begin{tabular}{ccrr}
\hline El & $\lambda, \AA$ & $E_{\text {low }}$ & $\log g f$ \\
\hline Sr I & 4607.33 & .00 & .17 \\
Y II & 4883.69 & 1.08 & .03 \\
& 4900.12 & 1.03 & -.06 \\
& 5087.42 & 1.08 & -.28 \\
& 5119.12 & .99 & -1.25 \\
& 5200.41 & .99 & -.59 \\
& 5402.77 & 1.84 & -.56 \\
& 5473.39 & 1.74 & -.97 \\
& 5509.91 & .99 & -1.06 \\
Ba II & 4554.04 & .00 & .11 \\
& 5853.68 & .60 & -.85 \\
& 6496.91 & .60 & -.30 \\
La II & 4662.51 & .00 & -1.14 \\
& 4748.73 & .93 & -.46 \\
Ce II & 4486.91 & .30 & -.19 \\
& 4562.36 & .48 & .28 \\
& 4572.28 & .68 & .25 \\
& 4628.16 & .52 & .18 \\
& 5274.22 & 1.04 & .29 \\
& 5472.28 & 1.25 & -.23 \\
Nd II & 5092.81 & .38 & -.79 \\
& 5234.21 & .55 & -.54 \\
& 5276.86 & .86 & -.75 \\
& 5319.81 & .55 & -.25 \\
Eu II & 6645.10 & 1.38 & .17 \\
\hline
\end{tabular}

interpolations through $T_{\text {eff }}$ and $\log g$ within the grid of model atmospheres used (Kurucz 1993). The model metallicities were taken with the accuracy \pm 0.25 dex (the choice of this model parameter should not be very important for our stars, because it is hydrogen that is the main electron donor in metal-deficient atmospheres). We found that the change by \pm 0.25 dex in the model metallicity causes changes as small as 0.001 in the derived abundances.

All abundances in this paper have been derived using the LTE assumption, despite numerous examples of nonLTE departures in metal-poor stars discussed in the literature. For example, Magain (1989) reported such findings for Fe I and Mg I lines in two halo stars HD 19445 and HD 140283. However, in our analysis we did not see any systematic decrease of Fe abundances with increasing excitation potential $E_{\text {low }}$ for these two stars (see, for example, Fig. 13 for HD 19445). The effects of non-LTE for Fe and $\mathrm{Mg}$ lines were investigated by Gratton et al. (1999). Using 60-level model of the iron atom, they found negligible de- partures from the LTE in high gravity stars and slightly more pronounced effects in low gravity stars, which is evidently due to the less efficient thermalization by collisions in giants. Non-LTE corrections for Fe lines are very small in dwarfs, and only small corrections $(<0.1 \mathrm{dex})$ are expected for giant stars. The main non-LTE effect for $\mathrm{Mg}$ is overionization, but for high excitation lines these corrections are small in cool dwarfs $\left(T_{\text {eff }}<6000 \mathrm{~K}\right)$ and larger in warmer dwarfs $(\approx 0.15 \mathrm{dex})$. Corrections are larger also in giants, where collisions less efficiently compete with photoionization. To alleviate this effect in giant atmospheres, we used only high-excitation lines of $\mathrm{Mg}$ I. Besides, only three stars in our pool have $T_{\text {eff }}>6000 \mathrm{~K}$. Fulbright (2000) investigated the abundances of 168 metal-poor dwarfs and showed that effects of non-LTE on the analysis of Fe I lines are very small on average. Spectroscopicallydetermined surface gravities are quite close to those obtained from Hipparcos parallaxes. Non-LTE calculations for Ba were performed in the work by Mashonkina et al. (1999). They showed that corrections are small for subordinate lines $(<0.08 \mathrm{dex})$ and increase to 0.20 for the Ba II resonance line $\lambda 4554 \mathrm{~m} \AA$. Departures from the LTE get stronger with lower metallicity, depend on temperature and microturbulence but are insensitive to surface gravity and $E W$. However, the observed underabundance of Ba at the considered low metallicities is $1.0-1.5 \mathrm{dex}$, and this amount cannot be removed completely only by accounting for these corrections. Therefore, we consider our analysis to be quite robust against non-LTE effects.

The abundances of investigated elements with respect to the Sun $[\mathrm{El} / \mathrm{Fe}]$ are given in Table 4 . Solar abundances were calculated with the $\log g f(\mathrm{G}, \mathrm{K})$ values and the solar model of Kurucz (1993). Solar EWs for the adopted line list were measured by us in the spectra of the Moon taken from the ELODIE library (Soubiran et al. 1998). The abundances thus derived are in a good agreement with those given in the paper of Gurtovenko \& Kostyk (1989).

For the elemental abundances derived from lines of neutral atoms, the effective temperature uncertainty is the most important: the total errors are within the limits of 0.04 to 0.12 dex. For the abundances derived from ions, the errors in log $g$ are more significant: for neutron capture elements, the total errors are of the order of 0.2 dex. 
Table 4. Relative abundance for program stars

\begin{tabular}{|c|c|c|c|c|c|c|c|c|c|c|c|}
\hline $\mathrm{HD} / \mathrm{BD}$ & {$[\mathrm{Fe} / \mathrm{H}]$} & {$[\mathrm{Mg} / \mathrm{Fe}]$} & {$[\mathrm{Si} / \mathrm{Fe}]$} & {$[\mathrm{Ca} / \mathrm{Fe}]$} & {$[\mathrm{Sr} / \mathrm{Fe}]$} & {$[\mathrm{Y} / \mathrm{Fe}]$} & {$[\mathrm{Ba} / \mathrm{Fe}]$} & {$[\mathrm{La} / \mathrm{Fe}]$} & {$[\mathrm{Ce} / \mathrm{Fe}]$} & {$[\mathrm{Nd} / \mathrm{Fe}]$} & {$[\mathrm{Eu} / \mathrm{Fe}]$} \\
\hline 245 & -0.78 & 0.23 & 0.24 & 0.30 & -0.28 & -0.10 & 0.17 & 0.08 & -0.21 & 0.16 & 0.46 \\
\hline 2796 & -2.21 & 0.12 & 0.33 & 0.43 & 0.23 & -0.20 & -0.26 & 0.19 & -0.14 & 0.11 & 0.31 \\
\hline 3546 & -0.63 & 0.18 & 0.25 & 0.24 & -0.11 & -0.16 & 0.09 & -0.11 & -0.05 & 0.16 & 0.31 \\
\hline 3567 & -1.20 & -0.06 & 0.21 & 0.41 & 0.37 & 0.00 & 0.27 & - & 0.23 & 0.30 & - \\
\hline 4306 & -2.52 & 0.27 & - & - & - & -0.32 & -0.57 & - & 0.45 & - & - \\
\hline 5395 & -0.19 & -0.04 & 0.05 & 0.14 & - & 0.03 & 0.32 & -0.05 & 0.09 & 0.21 & 0.17 \\
\hline 5916 & -0.51 & 0.05 & 0.15 & 0.19 & -0.25 & -0.19 & 0.06 & - & -0.29 & -0.11 & 0.06 \\
\hline 6582 & -0.89 & 0.29 & 0.25 & 0.36 & -0.08 & 0.11 & 0.06 & 0.14 & -0.07 & 0.08 & - \\
\hline 6755 & -1.47 & 0.19 & 0.2 & 0.25 & -0.13 & -0.09 & -0.03 & -0.01 & -0.12 & 0.24 & 0.41 \\
\hline 6833 & -0.89 & 0.11 & 0.13 & 0.26 & -0.05 & -0.25 & -0.24 & -0.17 & -0.12 & 0.12 & 0.00 \\
\hline 8724 & -1.65 & 0.22 & 0.38 & 0.37 & -0.11 & -0.16 & 0.18 & 0.13 & -0.12 & 0.15 & 0.09 \\
\hline 10700 & -0.56 & 0.24 & 0.14 & 0.32 & 0.10 & -0.05 & 0.07 & - & -0.07 & 0.23 & - \\
\hline 13530 & -0.48 & 0.32 & 0.26 & 0.33 & -0.05 & -0.12 & 0.06 & 0.08 & 0.05 & 0.11 & - \\
\hline 13783 & -0.61 & 0.26 & 0.18 & 0.26 & -0.18 & -0.01 & -0.06 & 0.22 & -0.01 & 0.10 & - \\
\hline 15596 & -0.67 & 0.32 & 0.30 & 0.36 & 0.17 & 0.11 & 0.33 & 0.03 & 0.04 & 0.34 & - \\
\hline 18768 & -0.51 & 0.00 & 0.10 & 0.15 & -0.04 & -0.10 & 0.22 & 0.03 & -0.06 & 0.01 & 0.26 \\
\hline 19445 & -1.89 & 0.14 & 0.40 & 0.42 & - & -0.02 & -0.17 & - & - & - & - \\
\hline 23439 & -1.14 & 0.30 & 0.40 & 0.46 & 0.32 & 0.26 & 0.19 & 0.12 & 0.13 & - & - \\
\hline 25329 & -1.73 & 0.24 & 0.26 & 0.45 & 0.18 & 0.12 & 0.04 & - & 0.24 & - & - \\
\hline 26297 & -1.91 & 0.14 & 0.41 & 0.38 & -0.33 & -0.26 & -0.24 & -0.02 & -0.27 & 0.11 & 0.17 \\
\hline 37828 & -1.58 & 0.26 & 0.42 & 0.47 & 0.01 & 0.10 & 0.08 & 0.18 & 0.17 & 0.39 & 0.35 \\
\hline 44007 & -1.49 & 0.16 & 0.34 & 0.38 & 0.16 & -0.01 & 0.08 & 0.24 & -0.13 & 0.25 & 0.26 \\
\hline 45282 & -1.28 & 0.00 & 0.19 & 0.31 & -0.14 & 0.00 & -0.02 & - & 0.17 & 0.19 & - \\
\hline 46480 & -0.49 & 0.26 & 0.20 & 0.38 & 0.13 & 0.16 & 0.17 & 0.17 & -0.15 & - & - \\
\hline 51530 & -0.39 & -0.04 & 0.10 & 0.15 & -0.01 & 0.05 & 0.21 & 0.14 & 0.05 & 0.10 & 0.00 \\
\hline 63791 & -1.67 & 0.29 & 0.40 & 0.35 & -0.17 & 0.12 & 0.33 & 0.18 & -0.05 & 0.22 & 0.34 \\
\hline 64090 & -1.69 & 0.12 & 0.34 & 0.30 & 0.07 & 0.00 & -0.10 & - & 0.18 & - & - \\
\hline 64606 & -0.82 & 0.22 & 0.17 & 0.34 & -0.12 & -0.10 & -0.21 & -0.14 & -0.09 & 0.05 & 0.31 \\
\hline 76932 & -0.90 & 0.17 & 0.29 & 0.44 & 0.23 & 0.10 & 0.23 & - & 0.02 & 0.12 & - \\
\hline 84937 & -2.00 & 0.36 & - & 0.34 & - & -0.02 & -0.10 & - & - & - & - \\
\hline 87140 & -1.71 & 0.10 & 0.40 & 0.40 & 0.15 & 0.16 & 0.25 & 0.22 & 0.11 & - & - \\
\hline 88609 & -2.66 & 0.30 & - & - & - & -0.16 & -0.87 & - & 0.09 & 0.23 & - \\
\hline 88725 & -0.65 & 0.15 & 0.21 & 0.23 & -0.05 & 0.04 & 0.04 & - & 0.13 & - & - \\
\hline 94028 & -1.43 & 0.17 & 0.35 & 0.41 & - & 0.15 & 0.06 & - & 0.08 & - & - \\
\hline 103095 & -1.39 & 0.20 & 0.23 & 0.34 & -0.12 & 0.04 & 0.06 & - & 0.23 & 0.36 & - \\
\hline 105755 & -0.65 & 0.22 & 0.31 & 0.34 & -0.09 & -0.06 & -0.08 & 0.23 & 0.28 & 0.14 & 0.17 \\
\hline 108076 & -0.85 & 0.20 & 0.24 & 0.34 & -0.05 & 0.01 & 0.14 & 0.33 & 0.03 & 0.34 & 0.17 \\
\hline 108317 & -2.17 & 0.33 & 0.30 & 0.34 & -0.04 & -0.15 & -0.31 & - & 0.22 & 0.27 & - \\
\hline 110184 & -2.27 & 0.25 & 0.18 & 0.31 & -0.27 & -0.42 & -0.34 & - & -0.29 & 0.03 & 0.28 \\
\hline 114762 & -0.72 & 0.17 & 0.19 & 0.29 & -0.10 & -0.13 & 0.04 & -0.01 & -0.18 & -0.04 & - \\
\hline 117876 & -0.47 & - & 0.18 & 0.26 & -0.10 & -0.01 & -0.18 & 0.03 & 0.01 & - & - \\
\hline 122563 & -2.42 & - & 0.26 & 0.28 & - & -0.42 & -1.11 & - & - & - & - \\
\hline 122956 & -1.60 & 0.13 & 0.23 & 0.13 & -0.13 & -0.25 & 0.01 & 0.00 & -0.17 & 0.12 & 0.43 \\
\hline 124897 & -0.58 & 0.10 & 0.25 & 0.26 & -0.17 & -0.01 & -0.07 & -0.02 & -0.06 & 0.15 & 0.39 \\
\hline 127243 & -0.65 & 0.27 & 0.26 & 0.24 & -0.05 & -0.04 & 0.13 & -0.02 & -0.10 & 0.14 & 0.20 \\
\hline 132142 & -0.51 & 0.18 & 0.25 & 0.29 & 0.00 & -0.09 & -0.07 & - & 0.07 & 0.25 & - \\
\hline 134169 & -0.72 & 0.06 & 0.23 & 0.25 & -0.04 & -0.12 & - & 0.13 & 0.01 & 0.17 & 0.08 \\
\hline 140283 & -2.41 & 0.21 & - & - & - & -0.19 & -0.78 & - & - & - & - \\
\hline 150177 & -0.64 & 0.05 & 0.13 & 0.17 & - & 0.06 & 0.28 & -0.12 & -0.05 & - & - \\
\hline 157089 & -0.56 & 0.11 & 0.20 & 0.22 & -0.01 & -0.10 & 0.01 & -0.06 & 0.04 & 0.08 & - \\
\hline 159482 & -0.86 & 0.30 & 0.26 & 0.36 & -0.08 & 0.01 & 0.11 & -0.09 & -0.24 & 0.14 & - \\
\hline 160693 & -0.46 & 0.20 & 0.12 & 0.23 & -0.09 & -0.05 & -0.18 & - & 0.01 & -0.03 & - \\
\hline 165195 & -2.03 & 0.33 & 0.36 & 0.36 & -0.10 & 0.05 & 0.05 & 0.14 & 0.10 & - & 0.35 \\
\hline 165908 & -0.61 & 0.01 & 0.09 & 0.23 & -0.04 & 0.00 & 0.13 & - & 0.13 & 0.00 & 0.16 \\
\hline 166161 & -1.20 & 0.20 & 0.44 & 0.35 & 0.21 & 0.09 & 0.37 & 0.13 & 0.12 & 0.19 & 0.21 \\
\hline 175305 & -1.42 & 0.08 & 0.30 & 0.31 & -0.14 & -0.07 & -0.14 & 0.19 & -0.09 & 0.29 & 0.20 \\
\hline 184499 & -0.64 & 0.18 & 0.29 & 0.31 & -0.07 & -0.01 & - & 0.06 & -0.12 & - & - \\
\hline
\end{tabular}


Table 4. continued

\begin{tabular}{|c|c|c|c|c|c|c|c|c|c|c|c|}
\hline $\mathrm{HD} / \mathrm{BD}$ & {$[\mathrm{Fe} / \mathrm{H}]$} & {$[\mathrm{Mg} / \mathrm{Fe}]$} & {$[\mathrm{Si} / \mathrm{Fe}]$} & {$[\mathrm{Ca} / \mathrm{Fe}]$} & {$[\mathrm{Sr} / \mathrm{Fe}]$} & {$[\mathrm{Y} / \mathrm{Fe}]$} & {$[\mathrm{Ba} / \mathrm{Fe}]$} & {$[\mathrm{La} / \mathrm{Fe}]$} & {$[\mathrm{Ce} / \mathrm{Fe}]$} & {$[\mathrm{Nd} / \mathrm{Fe}]$} & {$[\mathrm{Eu} / \mathrm{Fe}]$} \\
\hline 187111 & -1.74 & 0.29 & 0.35 & 0.40 & -0.07 & -0.11 & -0.14 & -0.16 & 0.02 & 0.12 & 0.12 \\
\hline 188510 & -1.48 & 0.12 & 0.39 & 0.40 & - & -0.07 & -0.04 & - & 0.24 & - & - \\
\hline 189558 & -1.07 & 0.22 & 0.32 & 0.32 & 0.21 & 0.15 & 0.24 & - & 0.07 & -0.08 & - \\
\hline 194598 & -1.11 & 0.03 & 0.19 & 0.30 & -0.17 & -0.17 & -0.06 & - & -0.01 & - & - \\
\hline 195633 & -0.55 & -0.03 & 0.13 & 0.21 & 0.00 & 0.09 & 0.12 & 0.08 & 0.11 & 0.10 & 0.13 \\
\hline 201889 & -0.85 & 0.17 & 0.32 & 0.35 & 0.10 & 0.11 & 0.03 & 0.06 & 0.09 & 0.01 & - \\
\hline 201891 & -0.99 & 0.04 & 0.24 & 0.28 & -0.13 & -0.08 & -0.05 & - & 0.29 & 0.23 & - \\
\hline 204155 & -0.78 & 0.14 & 0.28 & 0.38 & -0.14 & 0.03 & -0.16 & 0.16 & -0.26 & 0.05 & 0.19 \\
\hline 204543 & -1.79 & 0.23 & 0.32 & 0.27 & -0.04 & -0.20 & 0.21 & -0.14 & -0.18 & 0.03 & 0.32 \\
\hline 208906 & -0.71 & -0.01 & 0.12 & 0.06 & -0.12 & 0.00 & 0.01 & 0.08 & -0.06 & 0.15 & 0.31 \\
\hline 216143 & -2.11 & 0.06 & 0.38 & 0.44 & 0.08 & -0.13 & -0.10 & 0.30 & -0.06 & 0.19 & 0.40 \\
\hline 216174 & -0.56 & 0.18 & 0.29 & 0.20 & -0.05 & 0.08 & 0.08 & 0.03 & -0.08 & 0.27 & - \\
\hline 218502 & -1.72 & 0.08 & 0.33 & 0.23 & - & 0.13 & 0.29 & - & - & - & - \\
\hline 218857 & -1.84 & 0.00 & 0.25 & - & - & - & -0.25 & - & 0.24 & 0.23 & - \\
\hline 219617 & -1.43 & 0.05 & 0.32 & 0.37 & - & -0.21 & -0.06 & - & 0.04 & 0.20 & - \\
\hline 221170 & -2.05 & 0.22 & 0.35 & 0.43 & -0.11 & -0.06 & 0.35 & 0.20 & 0.10 & 0.32 & - \\
\hline 221377 & -0.88 & 0.02 & 0.26 & 0.17 & -0.06 & -0.03 & -0.08 & 0.03 & -0.15 & - & 0.14 \\
\hline 224930 & -0.85 & 0.30 & 0.21 & 0.44 & 0.03 & 0.12 & 0.03 & 0.14 & -0.18 & 0.10 & 0.22 \\
\hline 338529 & -2.31 & - & - & - & - & - & 0.34 & - & - & - & - \\
\hline 345957 & -1.33 & 0.08 & 0.21 & 0.33 & 0.25 & 0.04 & 0.36 & - & 0.35 & 0.34 & - \\
\hline-185550 & -3.01 & 0.28 & - & - & - & - & -1.12 & - & - & - & - \\
\hline+023375 & -2.26 & 0.09 & - & - & - & 0.01 & -0.31 & - & - & - & - \\
\hline+024651 & -1.82 & 0.19 & - & 0.31 & - & -0.30 & 0.12 & - & - & - & - \\
\hline+044551 & -1.51 & 0.19 & 0.37 & 0.36 & - & 0.09 & 0.43 & - & 0.28 & - & - \\
\hline+174708 & -1.56 & 0.04 & 0.41 & 0.51 & - & -0.04 & -0.25 & - & - & - & - \\
\hline+233130 & -2.62 & 0.19 & - & - & 0.17 & -0.32 & -0.46 & - & 0.04 & - & - \\
\hline+290366 & -1.01 & 0.20 & 0.28 & 0.29 & -0.12 & -0.07 & -0.06 & 0.20 & 0.01 & - & - \\
\hline +292091 & -1.93 & 0.01 & 0.36 & - & - & -0.02 & -0.13 & - & - & - & - \\
\hline+302611 & -1.41 & 0.16 & 0.16 & 0.32 & -0.37 & -0.21 & 0.07 & 0.07 & 0.16 & 0.37 & 0.49 \\
\hline+362165 & -1.51 & -0.01 & 0.21 & - & - & 0.10 & -0.08 & - & - & - & - \\
\hline +413931 & -1.68 & 0.06 & 0.30 & 0.40 & -0.01 & 0.12 & 0.20 & - & - & - & - \\
\hline+423601 & -1.97 & -0.09 & - & - & 0.24 & 0.35 & - & - & - & - & \\
\hline+660268 & -1.95 & - & 0.52 & 0.53 & - & - & 0.09 & - & - & - & - \\
\hline
\end{tabular}

\section{Results and discussion}

\section{1. $\alpha$-chain process elements}

Massive stars which explode as supernovae are responsible for most of the elements heavier than helium produced in the Galaxy. According to the fundamental paper by Burbidge et al. (1957), all known nuclear species are produced in separate processes. To carry out the correct analysis of $n$-capture element abundances, we also considered $\alpha$-element abundances. We investigated $\mathrm{Mg}, \mathrm{Si}, \mathrm{Ca}$, and excluded $\mathrm{Ti}$, which is sometimes referred to as an ironpeak element (Timmes et al. 1995), but whose overabundance at low metallicities follows the $\alpha$-pattern behaviour. In Fig. 14 we plotted our abundances together with the tracks computed by Timmes et al. (1995) and by Pagel \& Tautvaišienè (1995) within the framework of their theories of the chemical evolution of our Galaxy. The thick solid line represents the results of Pagel \& Tautvaišienė (1995). The thin solid line corresponds to the data from Timmes et al. (1995), the dashed line shows variation of the iron yield by a factor of two and the dotted line reflects a variation in the exponent of the initial mass function by 0.3 . For $[\mathrm{Si} / \mathrm{Fe}]$ vs. $[\mathrm{Fe} / \mathrm{H}]$ and for $[\mathrm{Ca} / \mathrm{Fe}]$ vs. $[\mathrm{Fe} / \mathrm{H}]$ plots, ob- servations are in reasonable agreement with both models. $[\mathrm{Mg} / \mathrm{Fe}]$ vs. $[\mathrm{Fe} / \mathrm{H}]$ is in better accord with the model of Pagel \& Tautvaišienè (1995), as one can see from Fig. 14. The discrepancy between the tracks for $\mathrm{Mg}$ of the two models is evident, and exist "for reasons which are not yet clear" (Pagel \& Tautvaišienè 1995). Both models assume that magnesium is a pure product of massive supernovae SNII. The best fit model of Timmes et al. (1995) to the $[\mathrm{Mg} / \mathrm{Fe}]$ observations may be a systematic reduction of the iron yields from massive stars by a factor of two and a small magnesium contribution originating from another source (Timmes et al. 1995). The mean values of $\alpha$-element abundances deduced in this work and the comparison with those obtained in other papers are given in Table 5 . The difference between $\langle[\alpha / \mathrm{Fe}]>$ obtained in various papers is within the errors of the determinations.

\section{2. n-capture elements}

The mean values of $n$-capture element abundances for our program stars are given in Table 6 . Understanding of the $s$-weak, $s$-main and and $r$-process relative contributions is crucial for the development of the modern chemical 
Table 5. Comparison of $\alpha$-element abundance determination

\begin{tabular}{|c|c|c|c|c|c|c|c|c|}
\hline El & $<[\mathrm{El} / \mathrm{Fe}]>$ & $\sigma$ & $<[\mathrm{El} / \mathrm{Fe}]>$ & $\sigma$ & $<[\mathrm{El} / \mathrm{Fe}]>$ & $\sigma$ & $<[\mathrm{El} / \mathrm{Fe}]>$ & $\sigma$ \\
\hline & 1 & & 2 & & 3 & & 4 & \\
\hline $\mathrm{Mg}$ & 0.30 & 0.11 & 0.44 & - & 0.30 & 0.11 & 0.25 & 0.10 \\
\hline $\mathrm{Si}$ & 0.26 & 0.09 & 0.44 & - & 0.36 & 0.17 & - & - \\
\hline $\mathrm{Ca}$ & 0.31 & 0.09 & 0.44 & - & 0.24 & 0.12 & 0.15 & 0.07 \\
\hline
\end{tabular}

* 1 - this work; 2 - McWilliam et al. (1995); 3 - Pilachowski et al. (1996); 4 - Jehin et al. (1999).
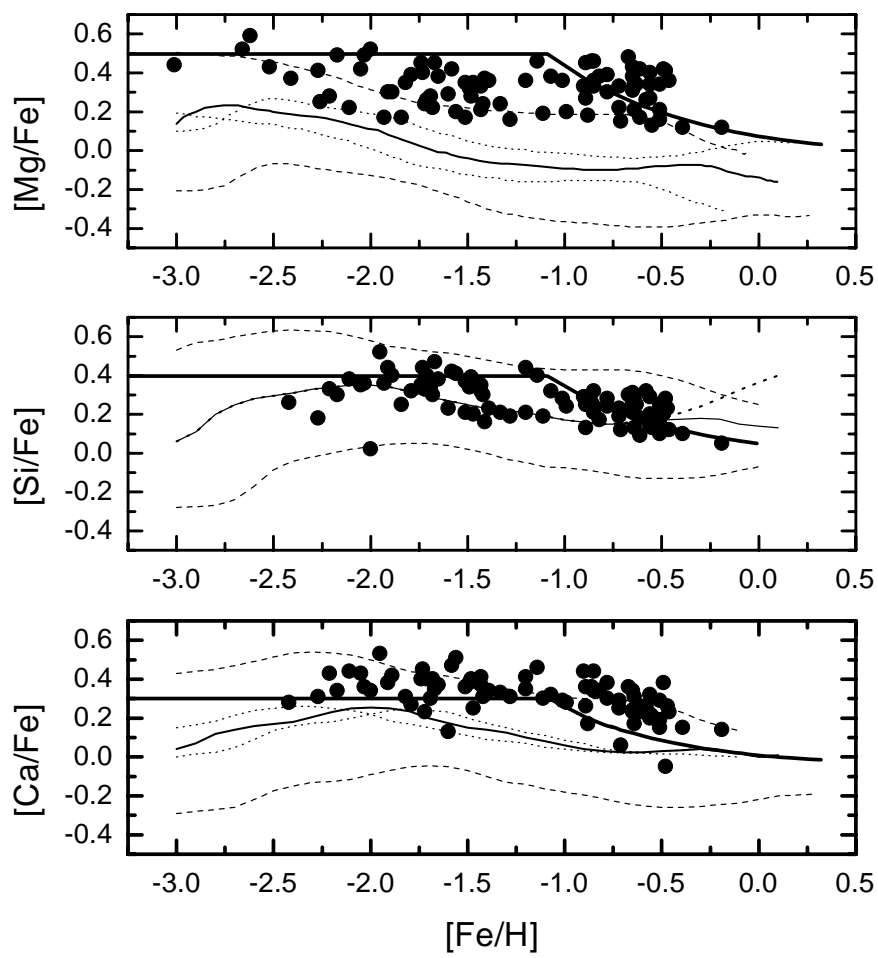

Fig. 14. Our relative abundances of the alpha-elements, $\mathrm{Mg}$, $\mathrm{Si}, \mathrm{Ca}$ and the tracks for these elements computed by Timmes et al. (1995) (thin solid line) and by Pagel \& Tautvaišienė (1995) (thick solid line)

evolution theories of our Galaxy. Investigation of these processes in the Solar System was carried out by Käppeler et al. (1989), Raiteri et al. (1991) and, more recently, by Arlandini et al. (1999). They conclude that more than $90 \%$ of Eu should come from the $r$-process. The study of the trends of relative abundances vs. $[\mathrm{Fe} / \mathrm{H}]$ is important to investigate the influence of the $n$-capture elements in the enrichment of the Galaxy. Relative abundances of $\mathrm{Sr}, \mathrm{Y}, \mathrm{Ba}, \mathrm{La}, \mathrm{Ce}$, and $\mathrm{Nd}$ to $\mathrm{Eu}$ may indicate the efficiency of the $s$-process at the epochs at which different metallicities were established (see Fig. 15). [Nd/Eu] exhibits larger contributions of the $r$-process than other elements. $[\mathrm{Y} / \mathrm{Eu}],[\mathrm{Ba} / \mathrm{Eu}]$ and $[\mathrm{Ce} / \mathrm{Eu}]$ show trends with $[\mathrm{Fe} / \mathrm{H}]$ that may be the evidence of the $s$-process enrichment growth with increasing metallicity. Mashonkina et al. (1999), by direct determination of the odd-to-even isotopic ratio from the Ba II resonance line, showed for two stars that they had been formed from a material whose barium content originated mainly in the $s$-process
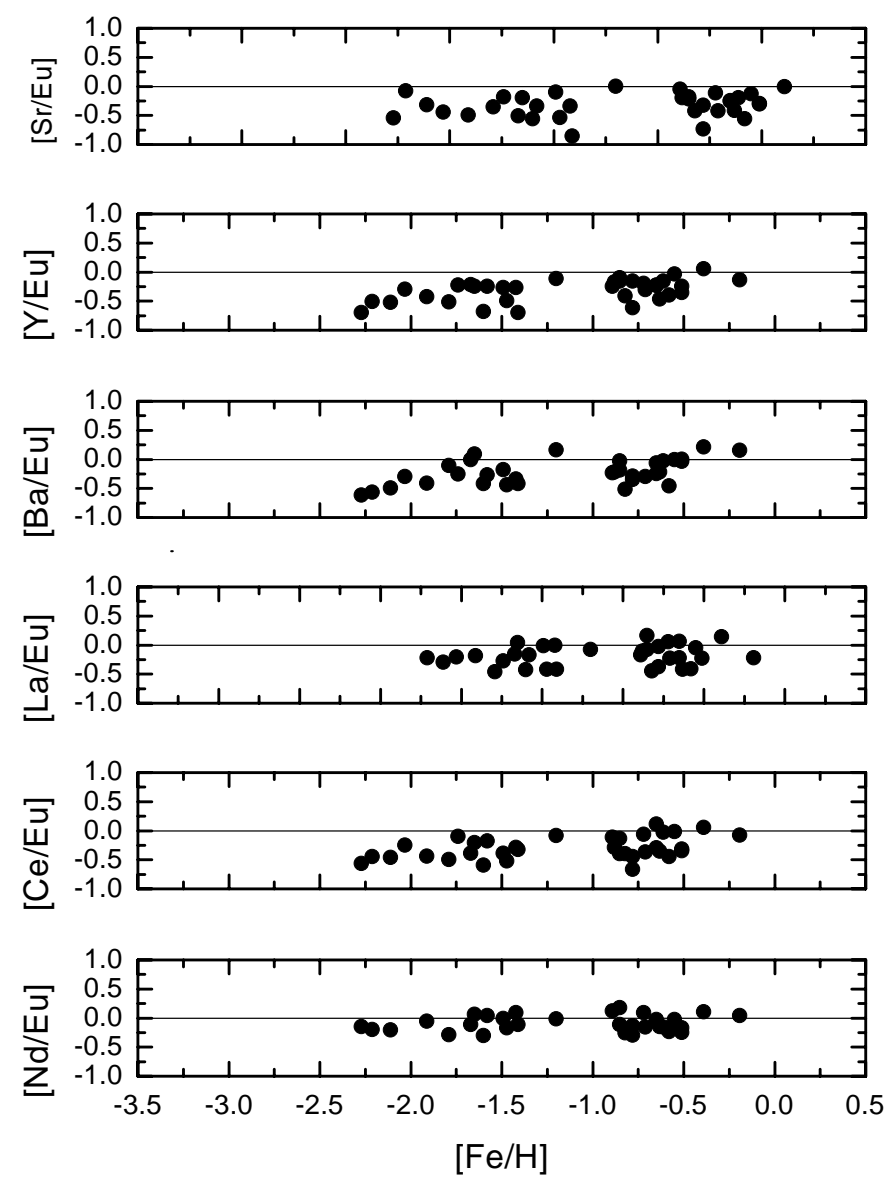

Fig. 15. Relative abundances of Sr, Y, Ba, La, Ce, Nd to Eu versus $[\mathrm{Fe} / \mathrm{H}]$.

$([\mathrm{Fe} / \mathrm{H}]>-2.2)$. This result agrees with our conclusion. The $[\mathrm{Ba} / \mathrm{Fe}]$ vs. $[\mathrm{Fe} / \mathrm{H}]$ plot, together with the Solar System $r$-process value (solid line, Arlandini et al. 1999), are given in Fig. 16. Only few stars near $[\mathrm{Fe} / \mathrm{H}]=-2.0$ follow the solar $r$-process pattern. Abundances relative to iron of $[\mathrm{Sr} / \mathrm{Fe}],[\mathrm{Y} / \mathrm{Fe}],[\mathrm{Ba} / \mathrm{Fe}],[\mathrm{La} / \mathrm{Fe}],[\mathrm{Ce} / \mathrm{Fe}],[\mathrm{Nd} / \mathrm{Fe}]$, $[\mathrm{Eu} / \mathrm{Fe}]$ vs. $[\mathrm{Fe} / \mathrm{H}]$ and comparisons with the tracks of the model from Pagel \& Tautvaišienė (1997) are given in Fig. 17. The run of $[\mathrm{Ba} / \mathrm{Fe}]$ vs. $[\mathrm{Fe} / \mathrm{H}]$ confirms the well-known jump of $\mathrm{Ba}$ abundances at $[\mathrm{Fe} / \mathrm{H}]$ about of -2.5 (Spite \& Spite 1978). Unfortunately, the lack of a large number of stars in our sample with metallicities $<-2.5$ does not permit us to trace the behaviour of Ba abundances at earlier times and check if there is a plateau at $[\mathrm{Fe} / \mathrm{H}]<-2.5$, as predicted by the model of 


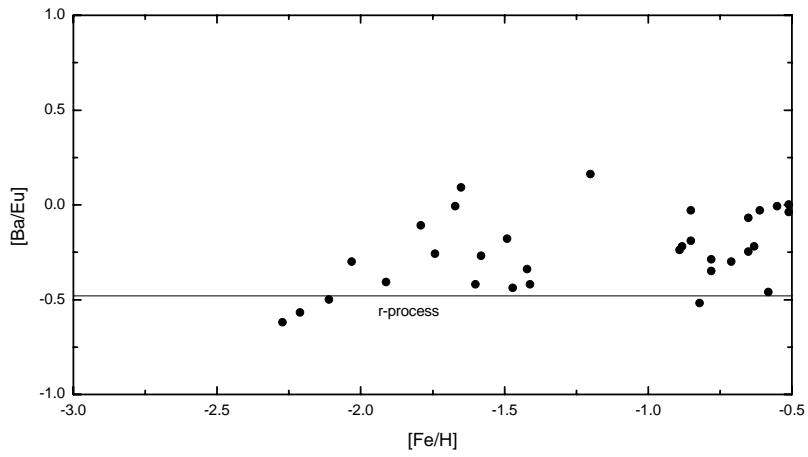

Fig. 16. $[\mathrm{Ba} / \mathrm{Fe}]$ vs. $[\mathrm{Fe} / \mathrm{H}]$ with Solar System $r$-process value (solid line, Arlandini et al. 1999)

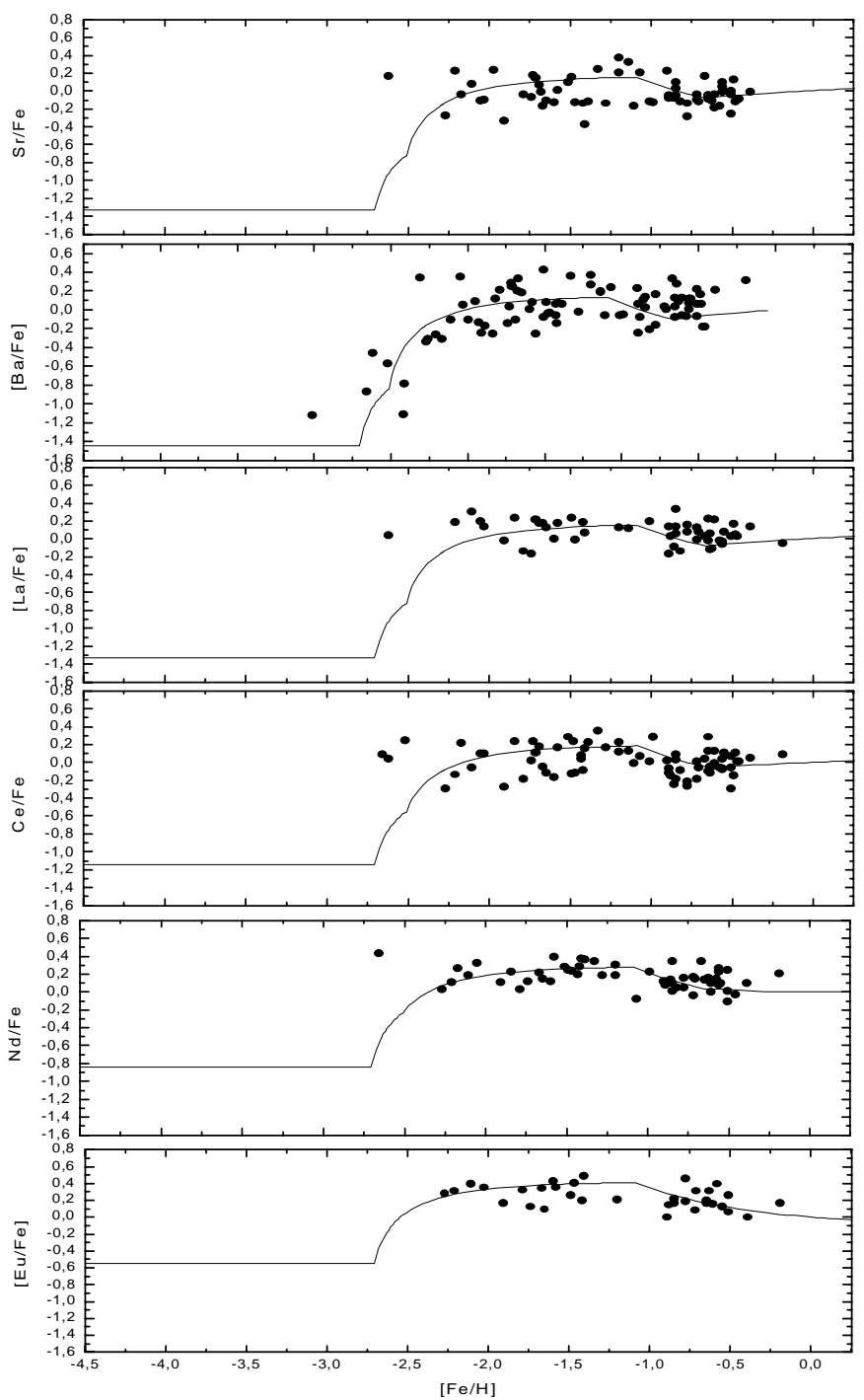

Fig. 17. Relative abundances of $\mathrm{Sr}, \mathrm{Y}, \mathrm{Ba}, \mathrm{La}, \mathrm{Ce}, \mathrm{Nd}$, and $\mathrm{Eu}$ versus $[\mathrm{Fe} / \mathrm{H}]$ and the tracks for these elements computed by Pagel \& Tautvaišienè (1997) (solid line)

Pagel \& Tautvaišienè (1997). However, inside the available range of metallicities $-0.5>[\mathrm{Fe} / \mathrm{H}]>-2.5$, agreement with these model calculations is quite close. As has been already mentioned, these theoretical abundances
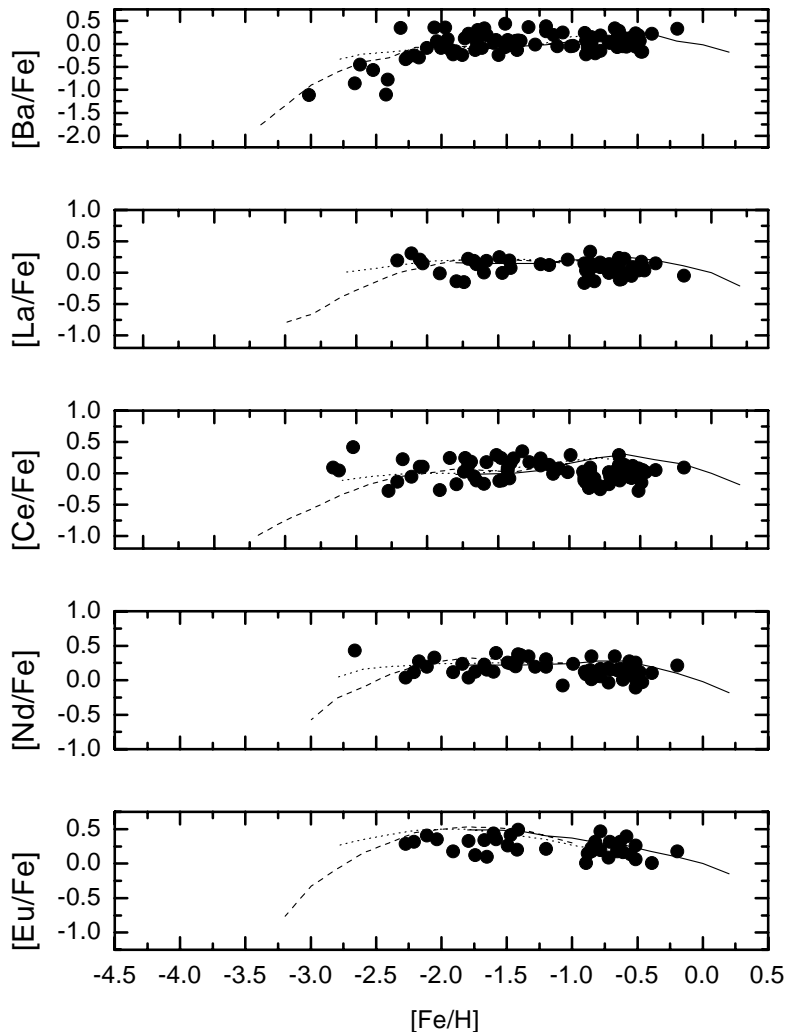

Fig. 18. Relative abundances of $\mathrm{Ba}, \mathrm{La}, \mathrm{Ce}, \mathrm{Nd}$, and $\mathrm{Eu}$ versus $[\mathrm{Fe} / \mathrm{H}]$ and the tracks for these elements computed by Travaglio et al. (1999) (the solid line corresponds to thin disk, the dotted line - thick disk, the dashed line - halo)

have been computed assuming two separate time scales for $s$-element production, of the order of $37 \mathrm{Myr}$ and 2.7 Gyr, corresponding to progenitor masses of about $8 M_{\odot}$ and $1.5 M_{\odot}$ respectively. Now, let's compare our results with the chemical evolution theory of Travaglio et al. (1999), which considers AGB stars of different masses and relatively low-mass type II SN as the production sites (Fig. 18) of $s$ - and $r$-elements. Ba, La, Ce, Nd, and $\mathrm{Eu}$, which contain species of very different origin (mostly $r$ process production for Eu and $s$-process for $\mathrm{Ba}$ ), were analyzed by these authors. They suppose that the $r$-process yield of $\mathrm{Ba}$ (for example) comes from 8-10 $M_{\odot}$ supernovae of type II and this component dominated in $\mathrm{Ba}$ abundances at low $[\mathrm{Fe} / \mathrm{H}]$. The $s$-contribution to Ba from AGB stars becomes dominant over the $r$-component after the first Gyr of galactic evolution (for $[\mathrm{Fe} / \mathrm{H}] \sim-1$ ). As remarked by Busso et al. (1999), this is linked both to the long time scales of low mass star evolution and to the efficiency of $s$-element production in AGB stars of different metallicities. Figure 18 represents the behaviour of these elements among the three populations of the Galaxy (the solid line corresponds to thin disk, the dotted line to the thick disk, the dashed line to the halo). For La, Nd, and $\mathrm{Eu}$ the agreement with observed data is good, while Ce and, especially, Ba show some scatter. It may be due to the fact that at $[\mathrm{Fe} / \mathrm{H}]<-1.5 \div-2.0$, the inhomogeneous models of chemical evolution are required. 
Table 6. The mean values of $n$-element abundance for program stars

\begin{tabular}{lrc}
\hline $\mathrm{El}$ & $<[\mathrm{El} / \mathrm{Fe}]>$ & $\sigma$ \\
\hline $\mathrm{Sr}$ & -0.03 & 0.15 \\
$\mathrm{Y}$ & -0.04 & 0.14 \\
$\mathrm{Ba}$ & -0.02 & 0.29 \\
$\mathrm{La}$ & 0.07 & 0.12 \\
$\mathrm{Ce}$ & 0.02 & 0.16 \\
$\mathrm{Nd}$ & 0.16 & 0.12 \\
$\mathrm{Eu}$ & 0.24 & 0.13 \\
\hline
\end{tabular}

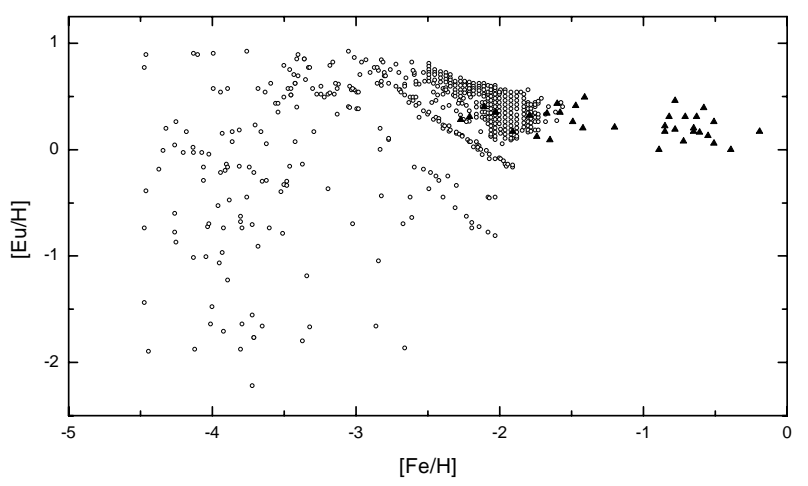

Fig. 19. $[\mathrm{Eu} / \mathrm{Fe}]$ versus $[\mathrm{Fe} / \mathrm{H}]$, the filled triangles represent our data, the small open circles correspond schematically to the calculations of Travaglio et al. (2001a). The $r$-process yields of $\mathrm{Eu}$ are derived from SN II in the mass range 8-10 $M_{\odot}$

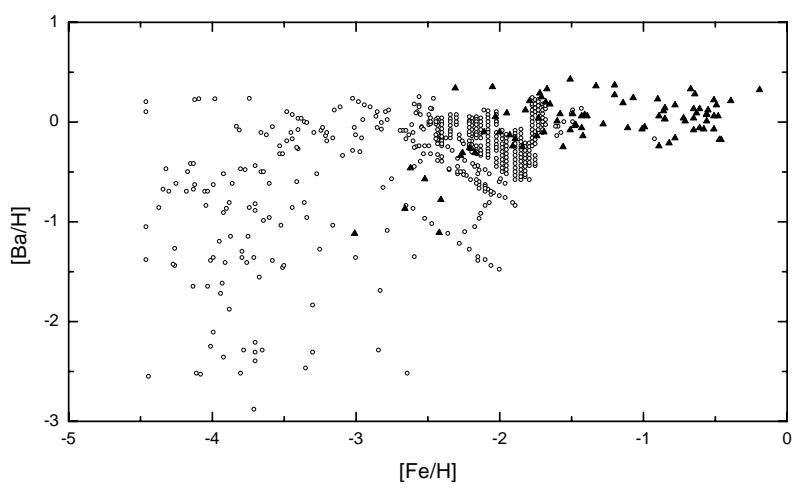

Fig. 20. The same of Fig. 19, for $[\mathrm{Ba} / \mathrm{Fe}]$ versus $[\mathrm{Fe} / \mathrm{H}]$

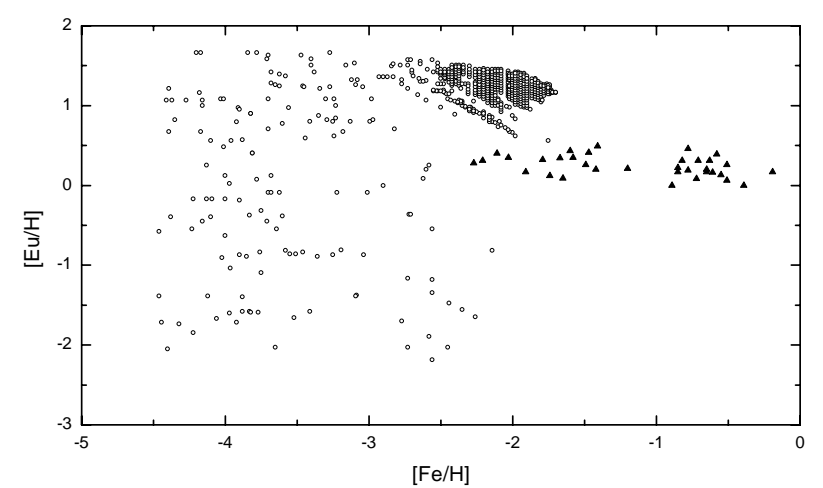

Fig. 21. $[\mathrm{Eu} / \mathrm{Fe}]$ versus $[\mathrm{Fe} / \mathrm{H}]$, the filled triangles represent our data, the small open circles correspond schematically to the calculations of Travaglio et al. (2001a). The $r$-process yields of Eu are derived from SN II in the mass range 15-30 $M_{\odot}$

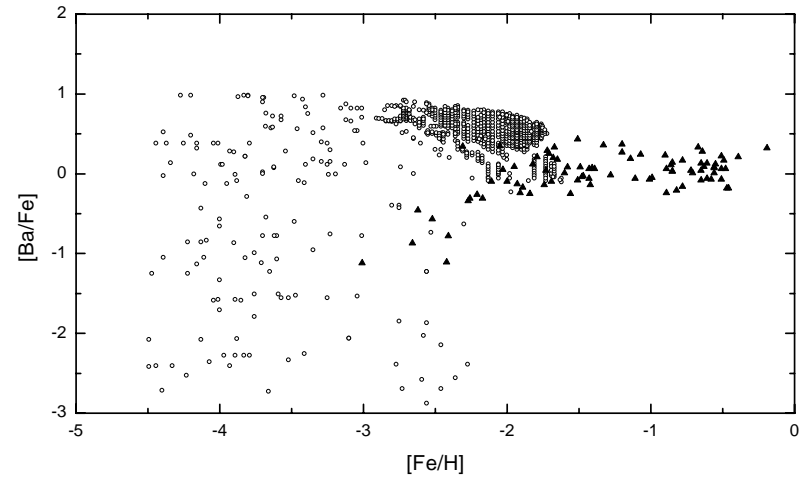

Fig. 22. The same of Fig. 21, for $[\mathrm{Ba} / \mathrm{Fe}]$ versus $[\mathrm{Fe} / \mathrm{H}]$

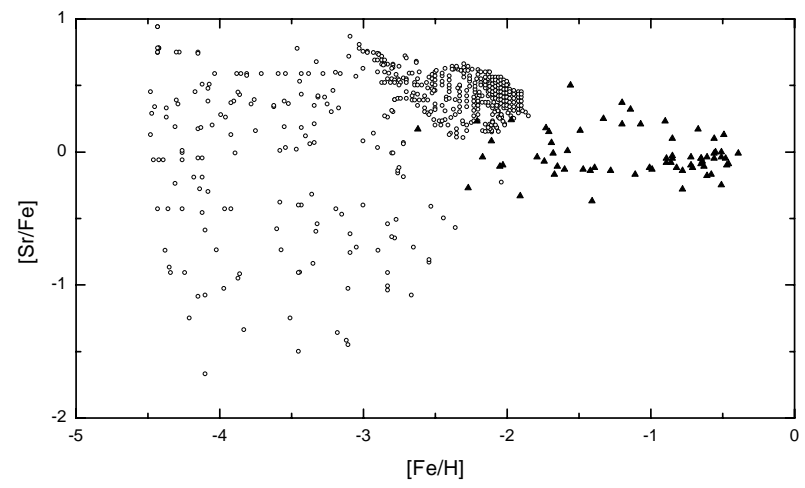

Fig. 23. $[\mathrm{Sr} / \mathrm{Fe}]$ versus $[\mathrm{Fe} / \mathrm{H}]$, the filled triangles represent our data, the small open circles correspond schematically to the calculations of Travaglio et al. (2001a)

Some of our target stars show that the $r$-process contributes to $\mathrm{Ba}, \mathrm{Y}$ and $\mathrm{Ce}$ abundances at $[\mathrm{Fe} / \mathrm{H}]<-2.0$. Sr shows larger scatter. McWilliam et al. (1995) and Ryan et al. (1996) found larger dispersion in $[\mathrm{Ba} / \mathrm{Fe}]$ and $[\mathrm{Sr} / \mathrm{Fe}]$ at lower metallicities $([\mathrm{Fe} / \mathrm{H}]<-2.5)$ and interpreted this as evidence of the formation of our Galaxy at early times by mergers of fragments with various proper enrichments (Searle \& Zinn 1968). Recently, inhomogeneous chemical evolution models have been constructed by Raiteri et al. (1999) for $[\mathrm{Ba} / \mathrm{Fe}]$ vs. $[\mathrm{Fe} / \mathrm{H}]$, by Travaglio et al. (2001a) for $[\mathrm{Eu} / \mathrm{Fe}],[\mathrm{Ba} / \mathrm{Fe}]$ and $[\mathrm{Sr} / \mathrm{Fe}]$ vs. $[\mathrm{Fe} / \mathrm{H}]$ and Tsujimoto et al. (2000) for $[\mathrm{Ba} / \mathrm{Mg}]$ vs. $[\mathrm{Mg} / \mathrm{H}],[\mathrm{Eu} / \mathrm{Mg}]$ vs. $[\mathrm{Mg} / \mathrm{H}]$. We compared our $[\mathrm{Eu} / \mathrm{Fe}],[\mathrm{Ba} / \mathrm{Fe}],[\mathrm{Sr} / \mathrm{Fe}]$ vs. $[\mathrm{Fe} / \mathrm{H}]$ data with the calculations of the inhomogeneous chemical evolution models by Travaglio et al. (2001a) (schematically, Figs. 19-23). Our data are overlapped only partially by the model dots in the common region of $[\mathrm{Fe} / \mathrm{H}]$. Figures 19-20 represent the abundances of $\mathrm{Eu}$ and $\mathrm{Ba}$ as the $r$-process yields from SNII in the mass range 8$10 M_{\odot}$. Figures 21, 22 show the same data as Figs. 19, 20, in which $\mathrm{Eu}$ and $\mathrm{Ba}$ are produced by SNII in the mass range $15-30 M_{\odot}$. Figure 23 displays chemical evolution of Sr with an additional primary component from SNII with masses $15-25 M_{\odot}$, accounting for $20 \%$ of the solar Sr abundance. As seen from Figs. 19 to 22, the models of chemical evolution with yields of Eu and Ba from low mass SNII reflect the observational data better. Nevertheless, 


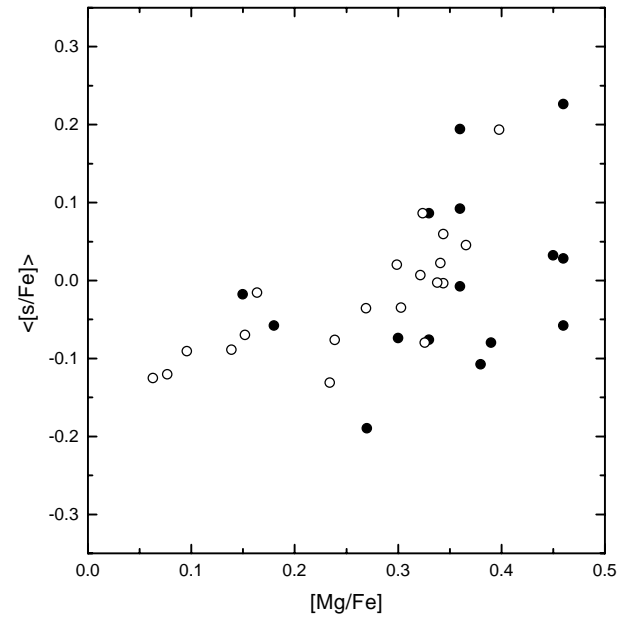

Fig. 24. Correlation diagram for $<[\mathrm{s} / \mathrm{Fe}]>$ versus $[\mathrm{Mg} / \mathrm{Fe}]$ in the region of $[\mathrm{Fe} / \mathrm{H}]=-1$

inhomogeneous models are very important for the interpretation of the behaviour of $r$ - and $s$-elements at early times of galactic evolution $($ at $[\mathrm{Fe} / \mathrm{H}]<-2.0)$.

\subsection{From globular clusters to field stars}

Jehin et al. (1999) suggested an interesting idea for the origin of metal-poor stars. They assumed that scatter in the $s$-process element abundances is caused by the different birth places of stars: all thick disk and field halo stars were born in globular clusters, from which they escaped either during an early disruption of the cluster (PopIIa) or, later, via evaporation processes (PopIIb). In our sample stars, the observed scatter in $s$-process element abundances $(1 \sigma \approx 0.15-0.20 \mathrm{dex})$ is larger than in $\alpha$-element abundances $(1 \sigma \approx 0.1 \mathrm{dex})$. To test this hypothesis, we selected stars from our target list with $[\mathrm{Fe} / \mathrm{H}]=-1 \pm 0.2$ dex and plotted $<[\mathrm{s} / \mathrm{Fe}]>$ vs. $[\mathrm{Mg} / \mathrm{Fe}]$ (see Fig. 24). Our data (filled circles) reproduce well the "Two branches diagram" (Jehin et al. 1999; open circles). A small shift (0.05 dex) in $[\mathrm{Mg} / \mathrm{Fe}]$ is due to the difference in the mean values of $[\mathrm{Mg} / \mathrm{H}]$ for two samples of stars. The correlation diagram for all our stars is given in Fig. 25. It is similar to that of Jehin et al. (1999, see their Fig. 19) with the overplotted observational data from Edvardsson et al. (1993). Two top dots at the "left" branch of our diagram have $[\mathrm{Fe} / \mathrm{H}]>-0.5$ dex. We also selected stars with $[\mathrm{Fe} / \mathrm{H}]=-2 \pm 0.2$ and constructed the same diagram of $<$ s $/ \mathrm{Fe}]>$ vs. $[\mathrm{Mg} / \mathrm{Fe}]$ (Fig. 26). We do not find any correlation between these parameters. Possible explanations of the lack of correlation are that at $[\mathrm{Fe} / \mathrm{H}] \sim-2 \div-2.2, s-$ process element abundances decrease so that the picture vanishes. Alternatively, the Jehin et al. (1999) scenario is suitable for stars with metallicities only about -1 . It should be noted that the larger dispersion of $s$-process element abundances may also be due to the fact that stars with $[\mathrm{Fe} / \mathrm{H}]=-1$ make up a mixed group of different populations, that have distinct pathways of $s$-process element enrichment.

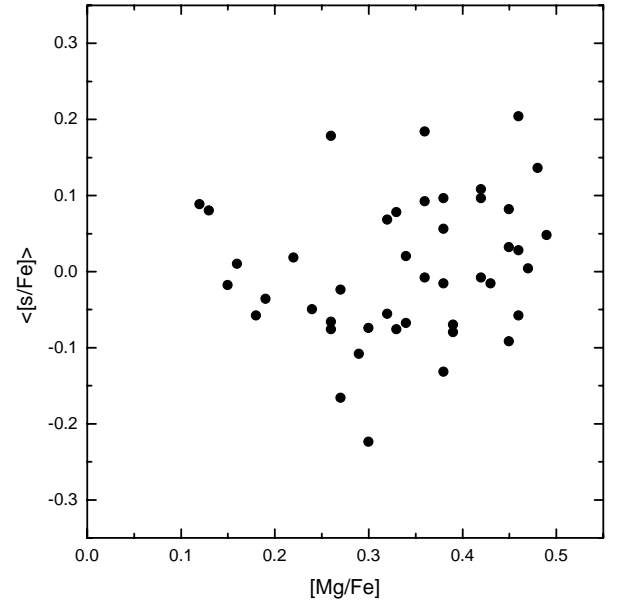

Fig. 25. Correlation diagram for $<[\mathrm{s} / \mathrm{Fe}]>$ versus $[\mathrm{Mg} / \mathrm{Fe}]$ for all our stars

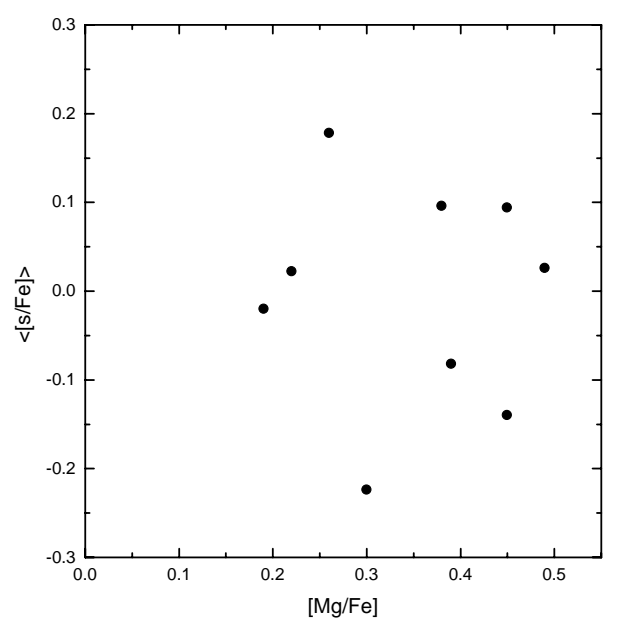

Fig. 26. Correlation diagram for $<[\mathrm{s} / \mathrm{Fe}]>$ versus $[\mathrm{Mg} / \mathrm{Fe}]$ in the region of $[\mathrm{Fe} / \mathrm{H}]=-2$

\section{Conclusions}

We derived model atmosphere parameters $\left(T_{\text {eff }}, \log g\right.$, $\left.[\mathrm{Fe} / \mathrm{H}], V_{\mathrm{t}}\right)$ for 90 metal-deficient stars $(-0.5<[\mathrm{Fe} / \mathrm{H}]$ $<-3)$, using eshelle spectra from the ELODIE library (Soubiran et al. 1998). These parameters were analyzed and compared with current determinations by other authors. The study of the following elements were carried out: $\mathrm{Mg}$, Si, Ca, Sr, Y, Ba, La, Ce, Nd and Eu. Relative contributions of $s$-and $r$-process elements were evaluated and interpreted by comparing them with the theoretical computations of chemical evolution models of the Galaxy. The runs of $[\mathrm{Si} / \mathrm{Fe}]$ and $[\mathrm{Ca} / \mathrm{Fe}]$ vs. $[\mathrm{Fe} / \mathrm{H}]$ were in good concordance with both chemical evolution theories of Timmes et al. (1995) and Pagel \& Tautvaišienè (1995). A discrepancy between the calculation of $[\mathrm{Mg} / \mathrm{Fe}]$ vs. $[\mathrm{Fe} / \mathrm{H}]$ and the one from the model of Timmes et al. (1995) was found. The trends of $n$-capture elements vs. metallicity are well described both by the model of Pagel \& Tautvaišienè $(1995 ; 1997)$ and by the model of Travaglio et al. (1999) at $[\mathrm{Fe} / \mathrm{H}]>-1.5$, when the matter of the Galaxy is sufficiently homogeneous. The analysis 
of $n$-capture element abundances confirms the jump in $[\mathrm{Ba} / \mathrm{Fe}]$ at $[\mathrm{Fe} / \mathrm{H}]=-2.5$. Frequently, this jump is interpreted as growth of the contribution of the $s$-process in enrichment of the interstellar medium (ISM). However, in view of models taking into account the current calculations of a yield of the $s$-process from AGB stars of different masses and metallicities, such behaviour of Ba may be conditioned predominantly by $r$-process yield from low mass SNII, up to $[\mathrm{Fe} / \mathrm{H}] \sim-1$. We note that the real origin of the $r$-process elements is under debate. There is a high uncertaintly in nuclear astrophysics and stellar nucleosynthesis calculations about the site where fast neutron captures occur. The solution of this question needs further development of the nucleosynthetic theories and $\mathrm{Ba}$ isotope composition studies in many stars of different metallicity. The larger (than for $\alpha$-element) dispersion for $s$-process element abundances may arise both from the birth of metal-poor stars in globular clusters with different evolution and (or) from different ways of $s$-element enrichment in Galaxy populations. The neutron capture element enrichment at $[\mathrm{Fe} / \mathrm{H}]<-2.0$ remains intriguing. Some stars from our sample at $[\mathrm{Fe} / \mathrm{H}]<-2.0$ show a large scatter for Sr, Ba, Y, Ce. Among metal-poor stars in the range of metallicities $-2.5<[\mathrm{Fe} / \mathrm{H}]<-4$, there are peculiar stars with excesses in $\mathrm{Sr}, \mathrm{Ba}$ and other elements, the intrinsic scatter of their abundances is quite significant (McWilliam et al. 1995; Ryan et al. 1996; McWilliam 1998; Sneden et al. 1998). Understanding of the $n$-capture enrichment at $[\mathrm{Fe} / \mathrm{H}]<-1.5$ relies on the current and future stochastic models of the early halo.

The main conclusions are as follows:

1. The chemical evolution models (Pagel \& Tautvaišiené 1995; Timmes et al. 1995) depict quite well the behaviour of $[\mathrm{Si} / \mathrm{Fe}],[\mathrm{Ca} / \mathrm{Fe}]$ with $[\mathrm{Fe} / \mathrm{H}]$. Two sources of $\mathrm{Si}$ and $\mathrm{Ca}$ production are considered in both models: high mass SN II (main contribution) and $\mathrm{SN}$ Ia. The trend of $[\mathrm{Mg} / \mathrm{Fe}]$ coincides better with the computations of Pagel \& Tautvaišiene (1995) than those of Timmes et al. (1995), despite the fact that both models assume one unique source of $\mathrm{Mg}$ enrichment - massive SN II;

2 . The runs of $n$-capture elements vs metallicity are well described both by the model of Pagel \& Tautvaišienè $(1995,1997)$ and by the model of Travaglio et al. (1999) at $[\mathrm{Fe} / \mathrm{H}]>-1.5$, when the matter of the Galaxy is sufficiently homogeneous;

3 . The analysis of $n$-capture element abundances confirms the jump in $[\mathrm{Ba} / \mathrm{Fe}]$ at $[\mathrm{Fe} / \mathrm{H}]=-2.5$;

4. At $[\mathrm{Fe} / \mathrm{H}]<-2.5 \div-2.8$, the essential contribution to the $n$-rich element abundances derives from the $r$-process. The main sources of these processes may be SN II;

5. The scatter in the $n$-capture elements is not due to errors in the determinations; it may reflect the inhomogeneous nature of the ISM at early stages of galactic evolution. It requires taking into account the formation of $n$-elements in individual nucleosynthetic events for early halo enrichment models;
6. The large dispersion of $n$-rich element abundances may be due both to the different contributions from the $s$ - and $r$-process, that appear at different epochs, and to a more inhomogeneous mixing of $r$-process nuclei with respect to $\alpha$-rich ones.

Acknowledgements. We are grateful to Dr. C. Soubiran and the research group at Haute Provence, who collected the library of ELODIE spectra. We wish to thank Dr. G. Tautvaišienè, who gave us the tracks of some elements, and N. Gorlova and Yu. Beletsky for some useful comments. We also thank Dr. M. Busso, the referee, for thoughtful advice which significantly improved this manuscript.

\section{References}

Alonso, A., Arribas, S., \& Martinez-Roger, C. 1996a, A\&A, 313,873

Alonso, A., Arribas, S., \& Martinez-Roger, C. 1996b, A\&AS, 117,227

Alonso, A., Arribas, S., \& Martinez-Roger, C. 1999a, A\&AS, 139,335

Alonso, A., Arribas, S., \& Martinez-Roger, C. 1999b, A\&AS, 140,261

Arlandini, C., Käppeler, F., Wisshak, K., Gallino, R., Lugaro, M., Busso, M., \& Straniero, O. 1999, ApJ, 525, 886

Axer, M., Fuhrmann, K., \& Gehren, T. 1995, A\&A, 300, 751

Burbidge, E. M., Burbidge, G. R., Fower, W. A., \& Hoyle, F. 1957, Rev. Mod. Phys., 29, 547

Burris, D. L., Pilachowski, A., Armandroff, T. E., et al. 2000, ApJ, in press

Busso, M., Gallino, R., \& Wasserburg, G. J. 1999, ARA\&A, 37,239

Cayrel de Strobel, G., Soubiran, C., Friel, E. D., Ralite, N., \& Francois, P. 1997, A\&AS, 124, 299

Cowan, J. J., Thielemann, F.-K, \& Truran, J. W. 1991, Phys. Rep., 208, 267

Edvardson, B., Andersen, J., Gustafsson, B., et al. 1993, A\&A, 275,101

Fuchs, B., Dettbarn, C., \& Wielen, R. 1994, in Ergodic Concepts in Stellar Dynamics, ed. P. Pfenninger, \& V. S. Gurzadyan (Springer-Verlag)

Fuhrmann, K., Axer, M., \& Gehren, T. 1994, A\&A, 285, 585

Fulbright, J. P. 2000, AJ, 120, 1841

Galazutdinov, G. A. 1992, Preprint SAO RAS, 92

Gallino, R., Busso, M., Lugaro, M., Travaglio, C., \& Straniero, O. 2000, 35th Liège Int. Astroph. Coll. The galactic halo: from globular clusters to field stars, ed. A. Noels, P. Magaine, D. Caro, et al., 81

Gilroy, K. K., Sneden, C., Pilachowski, C. A., \& Cowan, J. J. 1988, ApJ, 327, 298

Gratton, R. G., \& Sneden, C. 1994, A\&A, 287, 927

Gratton, R. G., Carretta, E., \& Castelli, F. 1996, A\&A, 314, 191

Gratton, R. G., Carretta, E., Eriksson, K., \& Gustafsson, B. 1999, A\&A, 350, 955

Gurtovenko, E. A., \& Kostyk, R. I. 1989, Fraunhoffer spectrum and system of solar Oscillators strengths, Naukova Dumka, Kiev, 200

Hollowell, D., Iben, I. Jr. 1989, ApJ, 340, 966

Iben, I. Jr., \& Renzini, A. 1982, ApJ, 259, L79

Jehin, E., Magain, P., Neuforge, C., et al. 1999, A\&A, 341, 241 
Käppeler, F., Beer, H., \& Wisshak, K. 1989, Rep. Prog. Phys., 52,945

Katz, D., Soubiran, C., Cayrel, R., Adda, M., \& Cautain, R. 1998, A\&A, 338, 151

King, J. R. 1993, AJ, 106, 1206

Kurucz, R. L. 1993, CD ROM n13

Lamb, S., Howard, W. M., Truran, J. W., \& Iben, I. Jr. , 1977, ApJ, 217, 213

Magain, P. 1989, A\&A, 209, 211

Malaney, R. A. 1987, ApJ, 321, 832

Mashonkina, L., Gehren, T., \& Bikmaev, I. 1999, A\&A, 343, 519

McWilliam, A., Preston, G. W., Sneden, C., \& Searle, L. 1995, AJ, 109, 2757

McWilliam, A. 1998, AJ, 115, 1640

Pagel, B. E. J., \& Tautvaišienè, G. 1995, MNRAS, 276, 505

Pagel, B. E. J., \& Tautvaišienè, G. 1997, MNRAS, 288, 108

Pilachowski, C. A., Sneden, C., \& Kraft, R. 1996, AJ, 111, 1689

Raiteri, C. M., Busso, M., Gallino, R., Picchio, G., \& Pulone, L. 1991, ApJ, 371, 665

Raiteri, C. M., Gallino, R., \& Busso, M. 1992, ApJ, 387, 263
Raiteri, C. M., Villata, M., Gallino, R., Busso, M., \& Cravanzola, A. 1999, ApJ, 518, L91

Ryan, S. G., Norris, J. E., \& Beers, T. C. 1996, ApJ, 471, 254

Searle, L., \& Zinn, R. 1978, ApJ, 225, 357

Sneden, C., Cowan, J. J., Burris, D. L., \& Truran, J. W. 1998, ApJ, 496, 235

Soubiran, C., Katz, D., \& Cayrel, R. 1998, A\&AS, 133, 221

Spite, M., \& Spite, F. 1978, A\&A, 67, 23

Thévenin, F., \& Idiart, T. 1999, ApJ, 521, 753

Timmes, F. X., Woosley, S. E., \& Weaver, T. A. 1995, ApJS, 98,617

Travaglio, C., Galli, D., Gallino, R., et al. 1999, ApJ, 521, 691

Travaglio, C., Burket, A., \& Galli, D. 2001a, ApJ, in press

Travaglio, C., Gallino, R., Busso, M., \& Gratton, R. 2001b, ApJ, in press

Truran, J. W. 1981, A\&A, 97, 391

Tsujimoto, T., Shigeyama, T., \& Yoshii, Y. 2000, 35th Liege Int. Astroph. Coll. The galactic halo: from globular clusters to field stars, ed. A. Noels, P. Magaine, D. Caro, et al., 51

Tsymbal, V. V. 1996, Model Atmospheres and Spectrum Synthesis, ASP Conf. Ser. 108, 198 